\title{
Mitochondrial $\mathrm{Na}^{+}$controls oxidative phosphorylation and hypoxic redox signalling
}

Pablo Hernansanz-Agustín ${ }^{1,2}$, Carmen Choya-Foces ${ }^{1}$, Susana Carregal-Romero ${ }^{3,4}$, Elena Ramos ${ }^{5}$, Tamara Oliva ${ }^{1}$, Tamara Villa-Piña ${ }^{5}$, Laura Moreno ${ }^{4,6}$, Alicia Izquierdo-Álvarez ${ }^{5}$, J. Daniel Cabrera-García ${ }^{1}$, Ana Cortés ${ }^{7,8}$, Ana Victoria Lechuga-Vieco ${ }^{2}$, Pooja Jadiya ${ }^{10}$, Elisa Navarro ${ }^{9}$, Esther Parada ${ }^{9}$, Alejandra Palomino-Antolín ${ }^{1,9}$, Daniel Tello $^{1}$, Rebeca Acín-Pérez ${ }^{2+}$, Juan Carlos Rodríguez-Aguilera ${ }^{7,8}$, Plácido Navas ${ }^{7,8}$, Ángel Cogolludo ${ }^{4,6}$, Iván LópezMontero $^{11}$, Álvaro Martínez-del-Pozo ${ }^{12}$, Javier Egea ${ }^{1,9}$, Manuela G. López ${ }^{9}$ John W. Elrod ${ }^{10}$, Jesús RuizCabello $^{3,4,13,14}$, Anna Bogdanova ${ }^{15}$, José Antonio Enríquez ${ }^{2,16^{*}}$, Antonio Martínez-Ruiz ${ }^{1,12,17^{*}}$

${ }^{1}$ Unidad de Investigación, Hospital Universitario Santa Cristina, Instituto de Investigación Sanitaria Princesa (IIS-IP), E-28009 Madrid, Spain

${ }^{2}$ Centro Nacional de Investigaciones Cardiovasculares Carlos III (CNIC), E-28029 Madrid, Spain

${ }^{3}$ Centro de Investigación Cooperativa en Biomateriales (CIC biomaGUNE), E-20014 San Sebastián, Spain.

${ }^{4}$ Centro de Investigación Biomédica en Red de Enfermedades Respiratorias (CIBERES), Spain

${ }^{5}$ Servicio de Inmunología, Hospital Universitario de La Princesa, IIS-IP, E-28006 Madrid, Spain.

${ }^{6}$ Departamento de Farmacología y Toxicología, Facultad de Medicina, Universidad Complutense de Madrid (UCM), IIS Gregorio Marañón (IISGM), E-28040 Madrid, Spain

${ }^{7}$ Centro Andaluz de Biología del Desarrollo, Universidad Pablo de Olavide-CSIC, E-41013 Sevilla, Spain

${ }^{8}$ Centro de Investigación Biomédica en Red de Enfermedades Raras (CIBERER), Spain

${ }^{9}$ Instituto Teófilo Hernando, Departamento de Farmacología y Terapéutica, Facultad de Medicina, Universidad Autónoma de Madrid (UAM), IIS-IP, E-28029 Madrid, Spain

${ }^{10}$ Center for Translational Medicine, Lewis Katz School of Medicine, Temple University, PA, 19140, USA.

${ }^{11}$ Departamento de Química Física, UCM, Instituto de Investigación Hospital “12 de Octubre” (i+12), E-28040, Madrid, Spain

${ }^{12}$ Departamento de Bioquímica y Biología Molecular, UCM, E-28040, Madrid, Spain.

${ }^{13}$ Ikerbasque, Basque Foundation for Science, 48013 Bilbao, Spain

${ }^{14}$ Departamento de Química en Ciencias Farmacéuticas, Facultad de Farmacia, UCM, E-28040, Madrid

${ }^{15}$ Inst. Veterinary Physiology, Vetsuisse Faculty and ZIHP, University of Zurich, CH-8057 Zurich, Switzerland

${ }^{16}$ Centro de Investigación Biomédica en Red de Fragilidad y Envejecimiento Saludable (CIBERFES), Spain

${ }^{17}$ Centro de Investigación Biomédica en Red de Enfermedades Cardiovasculares (CIBERCV), Spain

${ }^{+}$Present address: Department of Medicine and Department of Molecular and Medical Pharmacology, David Geffen School of Medicine at University of California, Los Angeles (UCLA), CA 90095-1735, Los Angeles, USA

* Address correspondence to: A.M.-R., Unidad de Investigación, Hospital Universitario Santa Cristina, IIS-IP C/ Maestro Vives 2, E-28009 Madrid (Spain) e-mail: amartinezruiz@salud.madrid.org, Tel.: +34-915574604; Fax: +34915202374, or J.A.E., CNIC, Melchor Fernández Almagro 3, E-28029 Madrid (Spain) e-mail: jaenriquez@cnic.es Tel.: +34-914531231 
All metazoans depend on $\mathrm{O}_{2}$ delivery and consumption by the mitochondrial oxidative phosphorylation (OXPHOS) system to produce energy. A decrease in $\mathrm{O}_{2}$ availability (hypoxia) leads to profound metabolic rewiring. In addition, OXPHOS uses $\mathrm{O}_{2}$ to produce reactive oxygen species (ROS) that can drive cell adaptations through redox signalling, but also trigger cell damage ${ }^{1-4}$, and both phenomena occur in hypoxia ${ }^{4-8}$. However, the precise mechanism by which acute hypoxia triggers mitochondrial ROS production is still unknown. $\mathrm{Ca}^{2+}$ is one of the best known examples of an ion acting as a second messenger ${ }^{9}$, yet the role ascribed to $\mathrm{Na}^{+}$is to serve as a mere mediator of membrane potential and collaborating in ion transport ${ }^{10}$. Here we show that $\mathrm{Na}^{+}$acts as a second messenger regulating OXPHOS function and ROS production by modulating fluidity of the inner mitochondrial membrane (IMM). We found that a conformational shift in mitochondrial complex I during acute hypoxia ${ }^{11}$ drives the acidification of the matrix and solubilization of calcium phosphate precipitates. The concomitant increase in matrix free$\mathrm{Ca}^{2+}$ activates the mitochondrial $\mathrm{Na}^{+} / \mathrm{Ca}^{2+}$ exchanger (NCLX), which imports $\mathrm{Na}^{+}$into the matrix. $\mathrm{Na}^{+}$interacts with phospholipids reducing IMM fluidity and mobility of free ubiquinone between complex II and complex III, but not inside supercomplexes. As a consequence, superoxide is produced at complex III, generating a redox signal. Inhibition of mitochondrial $\mathrm{Na}^{+}$import through NCLX is sufficient to block this pathway, preventing adaptation to hypoxia. These results reveal that $\mathrm{Na}^{+}$import into the mitochondrial matrix controls OXPHOS function and redox signalling through an unexpected interaction with phospholipids, with profound consequences in cellular metabolism.

Cells and tissues produce a superoxide burst as an essential feature of several adaptive responses, including hypoxia ${ }^{7,11}$. Given the importance of NCLX in ischemia-reperfusion injury ${ }^{12}$ we studied whether this exchanger may have a role in hypoxic redox signalling. Primary bovine aortic endothelial cells (BAECs) and mouse embryonic fibroblasts (MEFs) exposed to acute 
hypoxia showed an increase in cytosolic $\mathrm{Ca}^{2+}\left(\mathrm{Ca}^{2+}\right.$ cyto $)$ and a decrease in cytosolic $\mathrm{Na}^{+}\left(\mathrm{Na}^{+}\right.$cyto $)$ that was prevented by NCLX knock-down with siRNAs, genetic deletion, overexpression of a dominant negative form of NCLX (dnNCLX) or pharmacologic inhibition with CGP-37157 (Fig. 1a-e; Extended Data Figs. 1a-j and 2). Importantly this effect was rescued by expression of human NCLX in NCLX KO MEFs (Fig. 1d-e; Extended Data Figs. 1e-g). In agreement with the role of NCLX in mitochondrial $\mathrm{Ca}^{2+}$ handling ${ }^{12}$, the transient increase in mitochondrial $\mathrm{Ca}^{2+}$ after
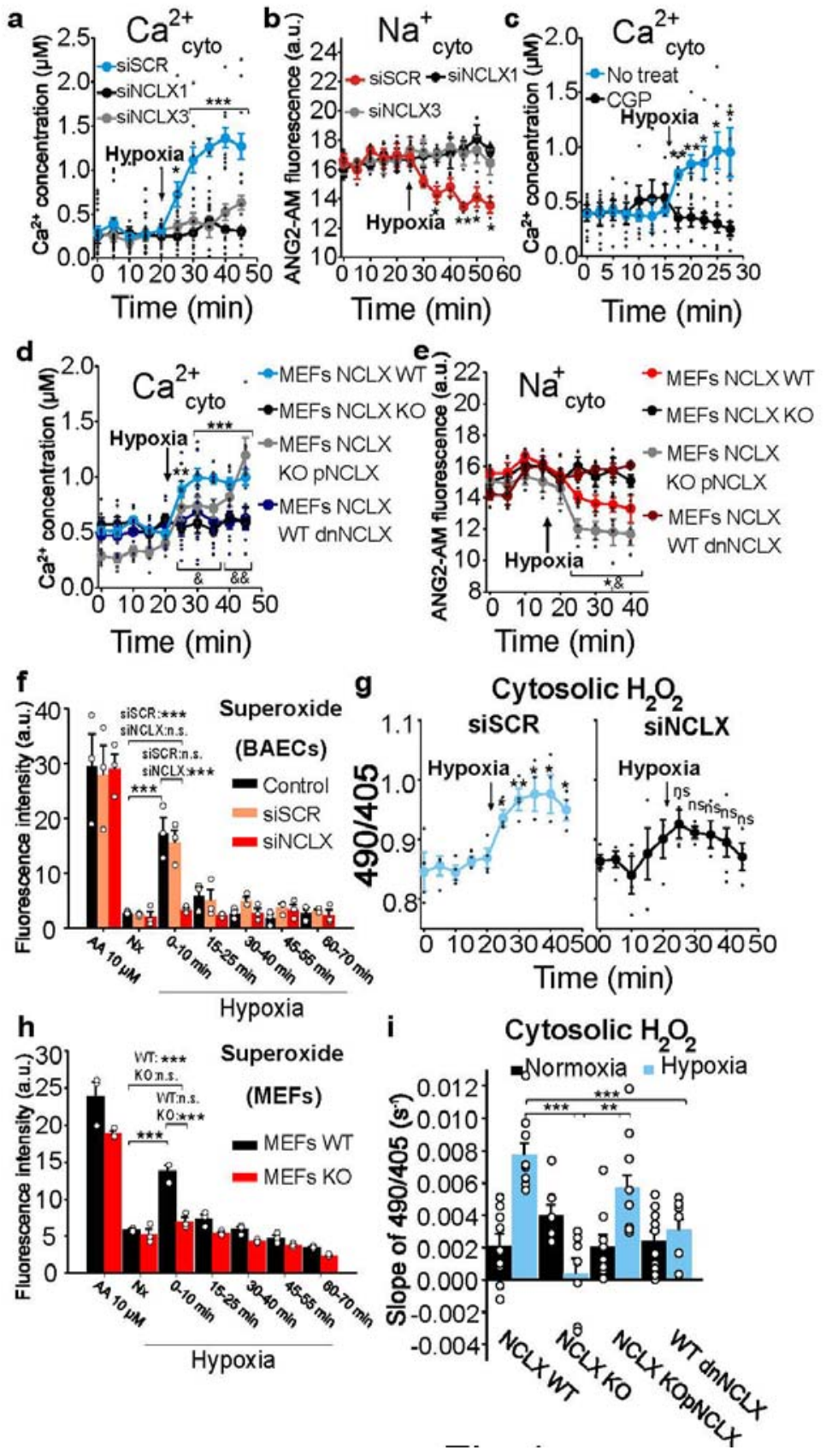

Fig. 1. Hypoxia activates $\mathrm{Na}^{+} / \mathrm{Ca}^{2+}$ exchange and enhances ROS production through NCLX. a-e, cytosolic $\mathrm{Ca}^{2+}$ (Cyto-GEM-GECO; $\mathrm{Ca}^{2+}$ cyto ) or $\mathrm{Na}^{+}$cyto (Asante Natrium 2-AM; ANG2-AM) measured by live cell confocal microscopy in normoxia and after induction of acute hypoxia $\left(1 \% \mathrm{O}_{2}\right)$. a-b, BAECs transfected with scramble siRNA (siSCR), siNCLX1 or siNCLX3. c, Non-treated BAECs (No treat) or treated with CGP-37157. d-e, WT, KO, $\mathrm{KO}+\mathrm{pNCLX}$ or WT+dnNCLX MEFs. f-i, ROS production measured by live cell confocal microscopy (g, i) or fixed cell fluorescence microscopy (f, h) in Nx or acute hypoxia $(1 \%$ $\mathrm{O}_{2}$ ). f, Superoxide detection after incubation with DHE in 10-min time windows in nontreated (Control), siSCR or siNCLX treated cells; $\mathrm{AA}=$ antimycin A. g, Detection of $\mathrm{H}_{2} \mathrm{O}_{2}$ with CytoHyPer in siSCR- or siNCLX-treated BAECs. h, Superoxide detection with DHE in WT or KO immortalized MEFs. i, Detection of $\mathrm{H}_{2} \mathrm{O}_{2}$ with CytoHyPer in WT, $\mathrm{KO}, \mathrm{KO}+\mathrm{pNCLX}$ or WT+dnNCLX immortalized MEFs. a-c, time-course traces of six independent experiments; d, e, time-course traces of four independent experiments; $\mathbf{f}, \mathbf{h}$, mean intensity of three independent experiments; g, time-course traces of four; $\mathbf{i}$, slopes of nine independent experiments. One-way ANOVA with Tukey's test for multiple comparisons (f-h, i) and student's t-test (last Nx time vs Hp times; a-e, g): * p < 0.05, ** p < 0.01, *** $\mathrm{p}<0.001$. Student's t-test (WT vs KO): ${ }^{\&} \mathrm{p}<0.05,{ }^{\text {\&\& }} \mathrm{p}<$ 0.01 . f,h, statistical comparisons shown only for $\mathrm{Nx}$ vs $0-10$ groups. 
histamine induction was more persistent upon NCLX down-regulation (Extended Data Fig. 1a-j). Notably, NCLX inhibition did not interfere with mitochondrial membrane potential or respiration (Extended Data Fig 1k-1). Thus, acute hypoxia induces NCLX activation.

The hypoxic ROS burst was blocked by NCLX knock-down by siRNA (from now on referred to as siNCLX), deletion, overexpression of dnNCLX, or inhibition with CGP-37157 (Fig.1f-i and Extended Data Fig. 3); and rescued by expression of human NCLX in KO MEFs (Fig. 1i). Therefore, NCLX activity is necessary for mitochondrial ROS production during hypoxia.

To date, mitochondrial complexes III (CIII) and I (CI) have been reported to be necessary for ROS production and cellular adaptation to hypoxia ${ }^{5,6,13}$. To examine their contribution, we knocked-down either CI subunit NDUFS4 or CIII subunit RISP, but only NDUFS4 knockdown abrogated $\mathrm{Na}^{+} / \mathrm{Ca}^{2+}$ exchange in hypoxia (Extended Data Fig. 4a-d). Accordingly, pharmacological inhibition of CI, but not of CIII or of complex IV (CIV), blunted hypoxiainduced $\mathrm{Na}^{+} / \mathrm{Ca}^{2+}$ exchange (Extended Data Fig. 4e-f) confirming that CI is necessary for NCLX activation in acute hypoxia.

Next, we evaluated how CI was influencing NCLX activity during hypoxia. CI presents two functionally different conformation states (A-CI and D-CI ${ }^{14-16}$, and we previously reported that the D-CI conformation, which is related to reduced CI activity ${ }^{14-16}$, is favoured in acute hypoxia $^{11}$. The D-CI conformation exposes Cys-39 of the ND3 subunit ${ }^{14}$ allowing fluorescent labelling at this residue for determination of conformation state ${ }^{11}$. Using this method, we confirmed that five min of hypoxia increased the D-CI conformation and, in parallel, we observed a decrease in the CI reactivation rate, regardless of NCLX inhibition (Extended Data Fig. 5a-d). Previously, we reported that hypoxia promotes a rotenone-sensitive matrix acidification ${ }^{11}$, which we also confirmed is independent of NCLX activity (Extended Data Fig. 5e). In addition, 
$\mathrm{Na}^{+} / \mathrm{Ca}^{2+}$ exchange was inhibited by rotenone (a CI inhibitor; Extended Data Fig. 5f-g), which further identifies D-CI formation as a necessary step in NCLX activation.

Acute hypoxia increased the matrix $\mathrm{Ca}^{2+}\left(\mathrm{Ca}^{2+}{ }_{\text {mito }}\right)$ concentration and this was augmented by NCLX knock-down (Fig. 2a). Thus, there is an increase in $\mathrm{Ca}^{2+}{ }_{\text {mito }}$ that drives NCLX activation and $\mathrm{Ca}^{2+}$ efflux to the cytosol. Intriguingly, BAECs treated with the mitochondrial $\mathrm{Ca}^{2+}$ uniporter (MCU) inhibitor Ru360 still displayed an increase in $\mathrm{Ca}^{2+}{ }_{\text {cyto }}$ and $\mathrm{Ca}^{2+}$ mito (Fig. 2b-c and Extended Data Fig.6a), excluding a role of $\mathrm{MCU}$ in this effect. We reasoned that if $\mathrm{Ca}^{2+}$ was not being introduced into the mitochondria, its source could be inside the organelles themselves. It has been known for decades that mitochondria harbour electron-dense spots composed by calcium phosphate precipitates which are extremely sensitive to acidification ${ }^{17}$, but whose function remains obscure ${ }^{18}$. We first confirmed by TEM-EDX analysis that the electron-dense spots that we found in the mitochondria were enriched in calcium, regardless of the element used to relativize (Extended Data Fig. 6b). Next, we confirmed that calcium phosphate precipitates decreased in both size and frequency in the presence of FCCP, a drug promoting strong acidification of the mitochondrial matrix, which concomitantly boosted $\mathrm{Ca}^{2+}{ }_{\text {mito }}$ content and elicited NCLX activation (Fig. 2d and Extended Data Fig. 6c-h). Indeed, acute hypoxia also promoted a decrease in the size of the precipitates, which correlated with an increase in soluble $\mathrm{Ca}^{2+}{ }_{\text {mito }}$ concentration (Fig. 2a, d and Extended Data Fig. 6c). Thus, mitochondrial calcium phosphate precipitates are the likely source of NCLX-dependent $\mathrm{Ca}^{2+}$ cyto increase. We have previously observed that pre-treatment of BAECs with the CI inhibitor rotenone promotes mitochondrial matrix acidification ${ }^{11}$. Since CI is involved in the hypoxic response, it could be related to the solubilization of mitochondrial $\mathrm{Ca}^{2+}$ precipitates through matrix acidification. We reasoned that if this was correct, pre-treatment with the CI-inhibitor rotenone should produce by itself matrix acidification and $\mathrm{Ca}^{2+}$ mito increase rendering mitochondria unable to respond to hypoxia. We pre-treated BAECs with rotenone for $30 \mathrm{~min}$ and subjected them to hypoxia, 
maintaining rotenone during the experiment. As hypothesized, $1 \mu \mathrm{M}$ rotenone blunted the increase in $\mathrm{Ca}^{2+}{ }_{\text {mito }}$ (Fig. 2c). At this point, we concluded that hypoxia induces the D-CI conformation, leading to mitochondrial matrix acidification and solubilization of calciumphosphate precipitates to liberate $\mathrm{Ca}^{2+}$ and activate NCLX.
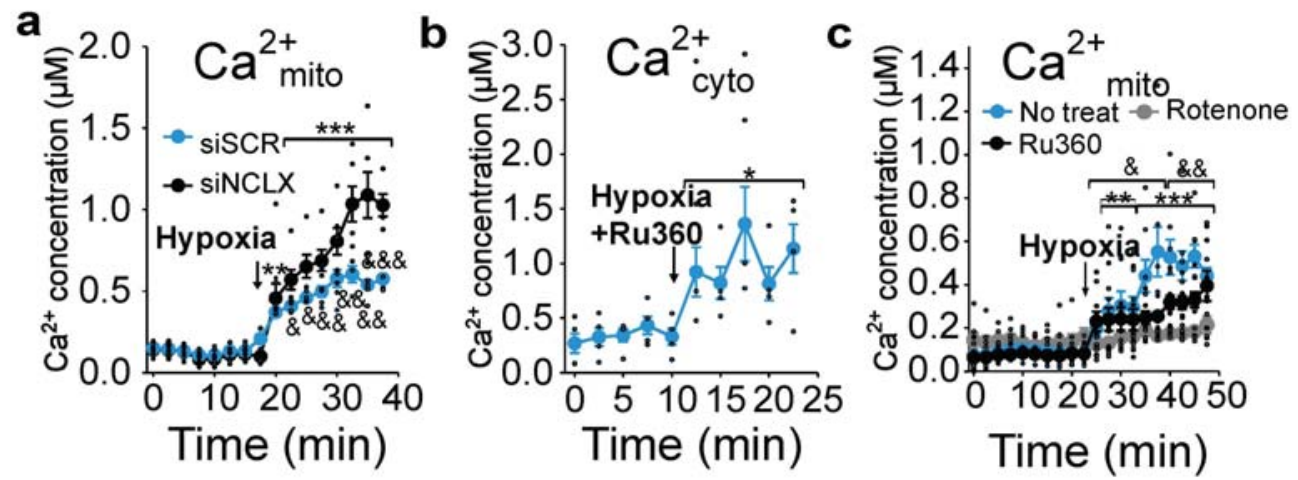

\section{CaP precipitates area}

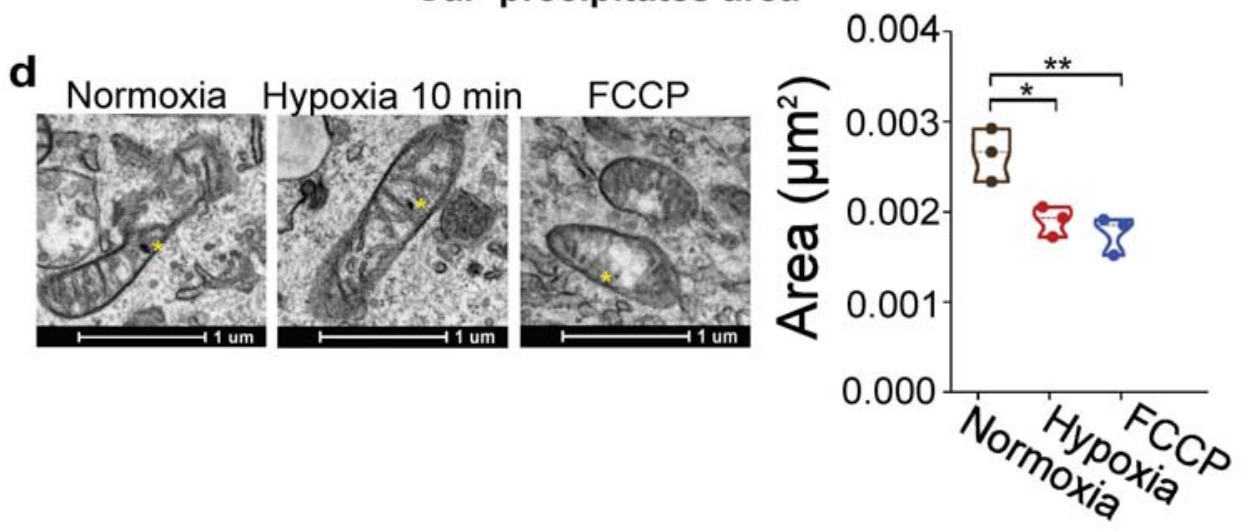

Fig. 2. Mitochondrial calcium phosphate precipitates are the source of mitochondrial $\mathrm{Ca}^{2+}$ raise and of NCLX activation. a, $\mathrm{Ca}^{2+}{ }_{\text {mito }}$ (Cepia $2 \mathrm{mt}$ ) measured by live cell confocal microscopy in BAECs transfected with siSCR or siNCLX during normoxia $(\mathrm{Nx})$ or acute hypoxia $\left(1 \% \mathrm{O}_{2}\right.$; trace of four independent experiments). $\mathbf{b}, \mathrm{Ca}^{2+}{ }_{\text {cyto }}$ measured by live cell confocal microscopy in BAECs subjected to normoxia $(\mathrm{Nx})$ and acute hypoxia $\left(1 \% \mathrm{O}_{2}\right)$ in the presence of $1 \mu \mathrm{M}$ Ru360, MCU blocker; trace of four independent experiments). c, $\mathrm{Ca}^{2+}{ }_{\text {mito }}$ measured by live cell confocal microscopy in non-treated BAECs or treated with $1 \mu \mathrm{M}$ Ru360 or $1 \mu \mathrm{M}$ rotenone during normoxia (Nx) or acute hypoxia ( $1 \% \mathrm{O}_{2}$; trace of five or more independent experiments). d, Representative TEM images and mean area of mitochondrial calcium phosphate precipitates during normoxia $\left(\mathrm{n}=110\right.$ precipitates), 10 min hypoxia $\left(1 \% \mathrm{O}_{2}\right.$; $\mathrm{n}=145$ precipitates) or $30 \mathrm{~min}$ treatment with $1 \mu \mathrm{M}$ FCCP ( $\mathrm{n}=96$ precipitates) in three independent experiments. Oneway ANOVA with Tukey's test for multiple comparisons (d) and student's t-test (last Nx time vs Hp times; a-c): * $\mathrm{p}<0.05,{ }^{* *} \mathrm{p}<0.01,{ }^{* * *} \mathrm{p}<0.001$. Student's t-test (siSCR vs siNCLX): ${ }^{*} \mathrm{p}<0.05,{ }^{\text {\&\& }} \mathrm{p}<0.01$, \&\&\& $\mathrm{p}<0.001$.

Next, we tested how NCLX activation triggered mitochondrial ROS production in acute hypoxia. In line with previous reports identifying CIII as a site of ROS production in hypoxia ${ }^{19,20}$, RISP knockdown abolished the superoxide burst in acute hypoxia (Fig. 3a) without affecting NCLX activation (Extended Data Fig. 4b-d). This effect was reproduced by myxothiazol, an 
inhibitor of CIII Qo site (Fig. 3b). ROS production by active isolated mitochondria transiently increased upon sequential addition of $\mathrm{Na}^{+}$and $\mathrm{Ca}^{2+}$, which activates $\mathrm{Na}^{+}$entry and $\mathrm{Ca}^{2+}$ extrusion through NCLX ${ }^{21,22}$ (Extended Data Fig. 7a).

The contribution of the different electron transport chain (ETC) complexes to respiration was measured in isolated mitochondria upon addition of different substrates and inhibitors ${ }^{23}$, in the absence or presence of $\mathrm{Na}^{+}$and $\mathrm{Ca}^{2+}$ to activate NCLX, with and without NCLX, or in the absence/presence of CGP-37157. In contrast to CIV-dependent respiration, CI- and CIIdependent respirations were decreased upon $\mathrm{NaCl}$ and $\mathrm{CaCl}_{2}$ addition, but only the reduction of CII-dependent respiration was dependent on NCLX activation (Fig. 3c-d and Extended Data Fig.7b-e). CII+III activity in isolated mitochondrial membranes was unaffected by the addition of $\mathrm{Ca}^{2+}$ (Extended Data Fig. 7f), but clearly decreased with $\mathrm{Na}^{+}$addition in a dose-dependent manner (Fig. 3e and Extended Data Fig.7g), which also increased ROS production (Fig. 3g). CII+III activity was decreased in mitochondrial membranes of WT MEFs subjected to hypoxia, an effect abolished by loss of NCLX (Fig. 3f). Dimethylmalonate (DMM, a complex II inhibitor) did not affect NCLX activity, but decreased the superoxide burst during hypoxia (Extended Data Fig. 7h-j). Surprisingly, individual CII or CIII activities showed no variation after $\mathrm{Na}^{+}$addition (Extended Data Fig. 7k-1); neither did CI+III activity (Fig. 3h). G3PD+III activity also decreased in the presence of $\mathrm{Na}^{+}$(Fig. 3i). In summary, while the individual activity of the different ETC complexes is not affected by $\mathrm{Na}^{+}$, the combined activities of different electron donors to $\mathrm{CoQ}$ with CIII behave differently based on the source of reducing equivalents, $\mathrm{FADH}_{2}(\mathrm{CII}$ and G3PDH) or NADH (CI).

Electron transfer between CII and CIII (and between G3PD and CIII) is limited by CoQ diffusion through the inner mitochondrial membrane, which depends on its fluidity ${ }^{24,25}$. We assessed whether IMM fluidity was affected by NCLX activation and $\mathrm{Na}^{+}$import during hypoxia. Acute hypoxia markedly increased mitochondrial $\mathrm{Na}^{+}\left(\mathrm{Na}^{+}{ }_{\text {mito }}\right)$ content through NCLX activation 
(Fig. 3j- k; Extended Data Fig. 7m-p). Employing fluorescence recovery after photobleaching (FRAP) of a red fluorescent protein targeted to the IMM (MitoRFP) we found that NCLX activation in acute hypoxia reduced membrane fluidity; this effect was abolished by NCLX inhibition by siRNA, genetic deletion, overexpression of dnNCLX, or CGP-37157 (Fig. 31-m, Extended Data Fig 8a and Supplementary videos 1-8). The FRAP signal was restored after ectopic expression of human NCLX in KO cells (Fig. 3m) suggesting direct causation. We also used the lipophilic quenchable fluorescent probe 10-n-nonyl acridine orange (NAO) that binds specifically to cardiolipin ${ }^{26}$, an abundant phospholipid of the $\mathrm{IMM}^{27,28}$. The reduced recovery rate of NAO fluorescence after photobleaching confirmed an NCLX-dependent reduction in IMM matrix side fluidity (Fig. 3n and Extended Data Fig. 8b). Reduced CoQ diffusion at the IMM matrix side would slow electron transfer from CIII to oxidized CoQ in the CIII Qi site during Q cycle turnover ${ }^{29}$. Accordingly, we observed that hypoxic NCLX activity promoted an increase in oxidized $\mathrm{CoQ}_{10}$ (Extended Data Fig. 8c). The fact that $\mathrm{Na}^{+}$exerts its effect when it is concentrated in the mitochondrial matrix highlights the physiological relevance of the asymmetric distribution of phospholipids between the matrix and the IMS leaflets of the mitochondrial inner membrane $\mathrm{IMM}^{28}$.

To further explore the mechanism by which $\mathrm{Na}^{+}$affects IMM fluidity, we measured anisotropy of fluorescent probes in phosphatydilcholine (PC) or PC:cardiolipin liposomes. In this cell free system, we observed that $\mathrm{Na}^{+}$addition (Fig. 3o and Extended Data Fig. 8d-e) reduced the lipid bilayer fluidity. The decrease in membrane fluidity caused by $\mathrm{Na}^{+}$was further supported by ESR experiments ${ }^{30}$, which suggested that $\mathrm{Na}^{+}-\mathrm{PC}$ interaction may occur at the level of phospholipid headgroups (Extended Data Fig. 8f-i). Infrared (IR) spectroscopy of PC liposomes treated with $\mathrm{NaCl}$ showed a distinct shift in the absorption peak of the PC's carbonyl group (Fig. $3 p$ and Extended Data Fig. 8j) and ICP mass spectrometry showed that the stoichiometry of the interaction is $0.29 \pm 0.04\left(\mathrm{Na}^{+}: \mathrm{PC}\right)$. These results demonstrate that $\mathrm{Na}^{+}$specifically interacts with 
phospholipids such as $\mathrm{PC}$ at the level of its carbonyl group, at a ratio of $3 \mathrm{PC}: 1 \mathrm{Na}^{+}$, what is in full agreement with previous estimations ${ }^{31,32}$, and suggests that this direct incorporation of $\mathrm{Na}^{+}$is responsible for reduced membrane fluidity during hypoxia.
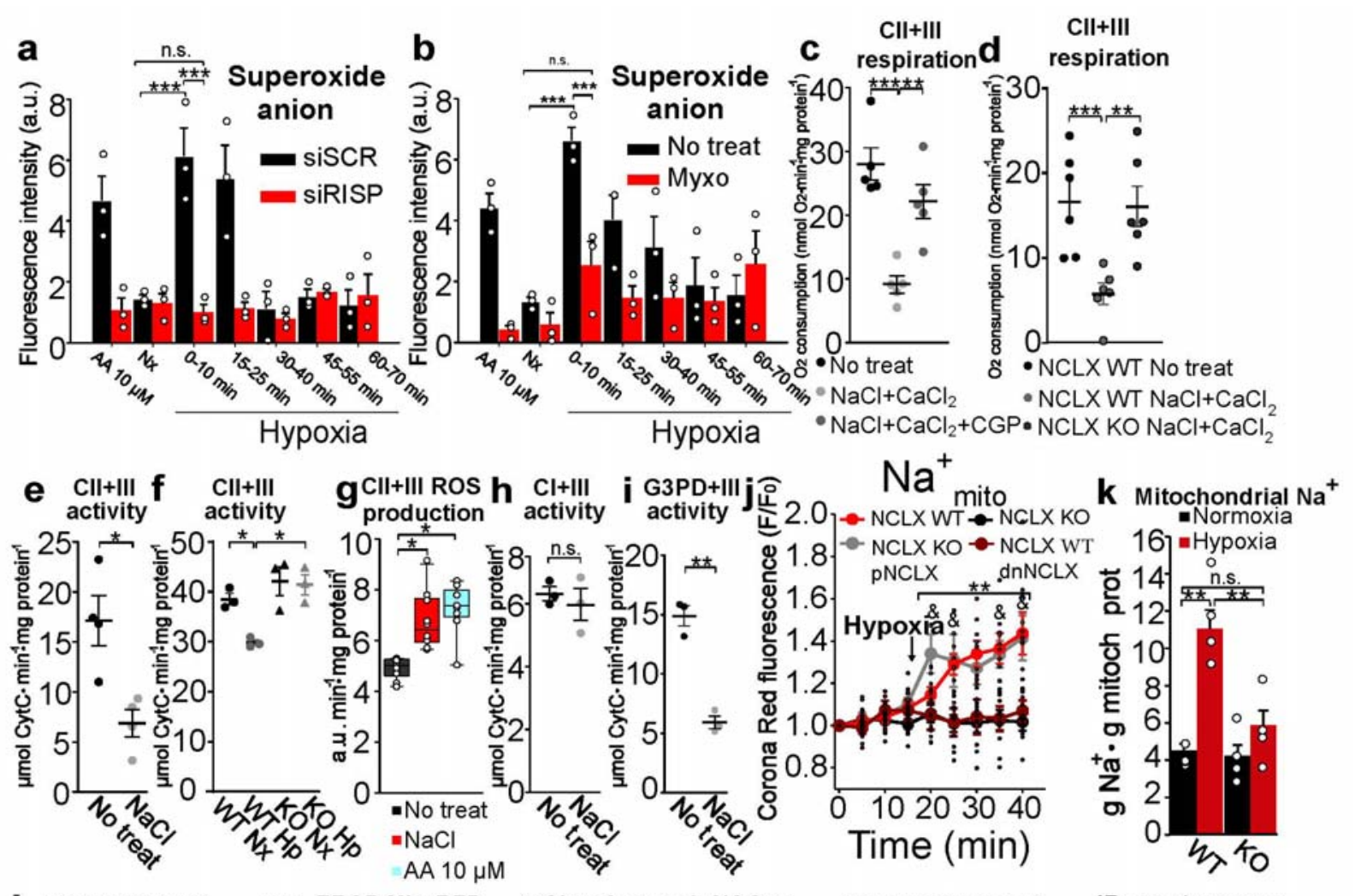

I FRAP MitoRFP m FRAP MitoRFP nSignal quench NAO O PC anisotropy $\mathbf{p}$ IR spectroscopy

-Normoxia"Hypoxia $\backsim$ Normoxia - Hypoxia $=$ Normoxia $=$ Hypoxia 0.074
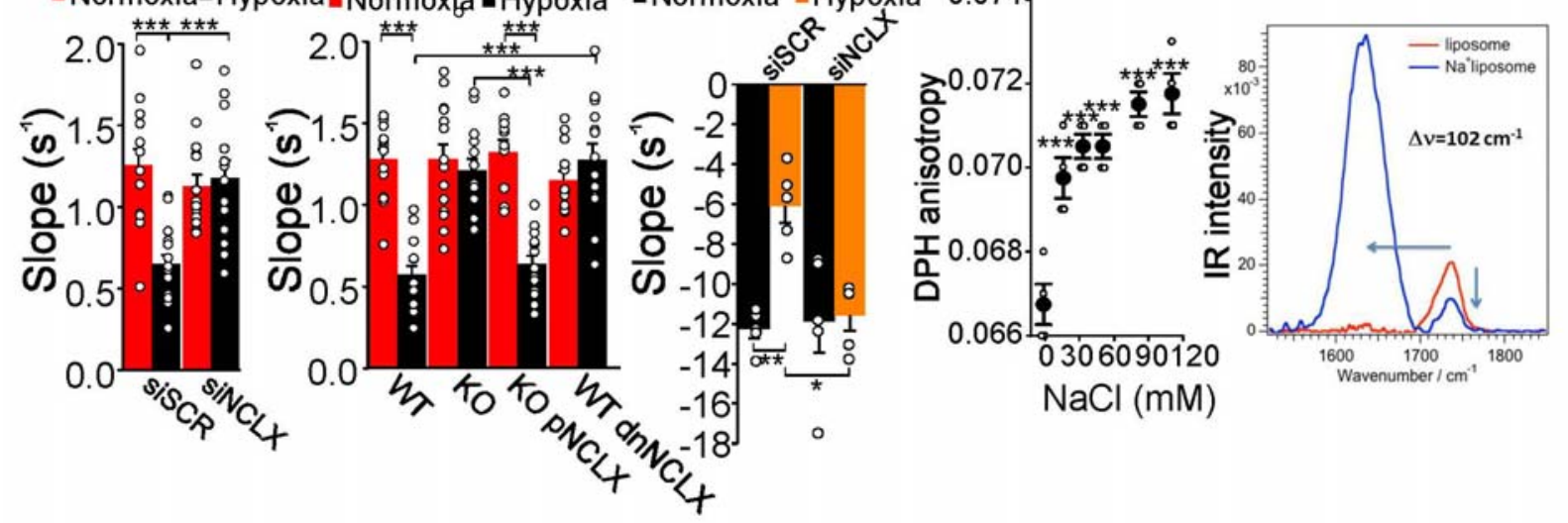

Fig. 3. NCLX governs superoxide production and OXPHOS function in hypoxia through $\mathrm{Na}^{+}$-dependent alteration of inner mitochondrial membrane fluidity. a-b, Superoxide detection with DHE in (a) siSCR, siRISP, (b) non-treated (No treat) or $1 \mu \mathrm{M}$ myxothiazol (Myxo) treated BAECs. c, d Effect of NCLX activation by $10 \mathrm{mM}$ $\mathrm{NaCl}$ and $0.1 \mathrm{mM} \mathrm{CaCl}_{2}$ on succinate-based OCR in isolated mitochondria from BAECs (c), $\mathrm{n}=5$, or MEFs (d), $\mathrm{n}=6$. e, Succinate-cytochrome $c$ activity in freeze-thawed mitochondria (i.e. mitochondrial membranes; MM) from BAECs $\pm 10 \mathrm{mM} \mathrm{NaCl}(\mathrm{n}=4)$. f, Succinate-cytochrome $c$ activity in MM from WT or KO MEFs previously subjected to normoxia $(\mathrm{Nx})$ or hypoxia $\left(\mathrm{Hp} ; 1 \% \mathrm{O}_{2} ; \mathrm{n}=3\right)$. g, ROS production on succinate-cytochrome c activity in MM from BAECs $\pm 10 \mathrm{mM} \mathrm{NaCl}, \mathrm{n}=10$. h, NADH-cytochrome $c$ activity in MM from BAECs $\pm 10 \mathrm{mM} \mathrm{NaCl}(\mathrm{n}=3)$. i, G3Pcytochrome $c$ activity in $\mathrm{MM}$ from BAECs $\pm 10 \mathrm{mM} \mathrm{NaCl}(\mathrm{n}=3)$. $\mathbf{j}-\mathbf{k}$, Effect of hypoxia $\left(1 \% \mathrm{O}_{2}\right)$ in $\mathrm{Na}^{+}{ }_{\text {mito }}$ content in WT and KO MEFs, measured with CoroNa-Green adapted for mitochondrial loading and live fluorescence $(\mathbf{j}, n=10)$, or with SBFI fluorescence after mitochondria isolation $(\mathbf{k}, \mathrm{n}=4)$. $\mathbf{l}-\mathbf{m}$, FRAP of siSCR or siNCLX BAECs $(\mathbf{l} ; \mathbf{n}=16)$ and $\mathrm{WT}, \mathrm{KO}, \mathrm{KO}+\mathrm{pNCLX}$ or $\mathrm{WT}+\operatorname{dnNCLX} \operatorname{MEFs}(\mathbf{m} ; \mathrm{n}=14)$ expressing mitoRFP in normoxia or hypoxia for 20 
$\min \left(1 \% \mathrm{O}_{2}\right)$. n, NAO FRAP quench signal of siSCR or siNCLX BAECs and exposed to normoxia or hypoxia for 15 $\min \left(1 \% \mathrm{O}_{2} ; \mathrm{n}=5\right)$. $\mathbf{0}$, Anisotropy of PC liposomes treated with increasing concentrations of $\mathrm{NaCl}$ measured by $\mathrm{DPH}$ fluorescence $(n=3)$. p, Infrared absorption spectra in the region of the carbonyl group of PC liposomes treated or not with $16 \mathrm{mM} \mathrm{NaCl}$. Student's t-test for pairwise comparisons and one-way ANOVA with Tukey's test for multiple comparisons. n.s. not significant, $* \mathrm{p}<0.05, * *<\mathrm{p} 0.01, * * * \mathrm{p}<0.001$. AA $=$ antimycin A. a,b, statistical comparisons shown only for Nx vs 0-10 groups.

Altogether, our data demonstrate that acute hypoxia promotes $\mathrm{CI} A / \mathrm{D}$ transition and mitochondrial matrix acidification ${ }^{11}$, which partially solubilizes mitochondrial calcium phosphate precipitates to increase matrix free $\mathrm{Ca}^{2+}$. The increase in matrix $\mathrm{Ca}^{2+}$ is then exported to the cytosol in exchange for $\mathrm{Na}^{+}$by NCLX. Matrix $\mathrm{Na}^{+}$directly binds IMM phospholipids and reduces membrane fluidity. This diminishes CoQ diffusion in the IMM matrix side and slows electron transfer between CII and CIII effectively uncoupling the Q cycle of CIII, increasing the half-life of the semiquinone form in the CIII Qo site, and promotes superoxide anion formation ${ }^{29}$.

To study the physiological relevance of this pathway, we investigated if its manipulation altered the response of pulmonary arteries to hypoxia. Rat pulmonary arteries (PA) exposed to hypoxia in the absence of pretone (precontraction) perform a rapid contractile response (peak contraction), which reaches a plateau (steady-state contraction). The latter is dependent on mitochondrial $\operatorname{ROS}^{33-35}$. We found that inhibition of mitochondrial $\mathrm{Na}^{+}{ }_{\text {mito }}$ entry by $\mathrm{NCLX}$ silencing inhibited the superoxide burst in rat pulmonary artery smooth muscle cells (PASMCs) subjected to acute hypoxia (Fig. 4a-b). Concomitantly, NCLX inhibition markedly reduced the steady-state component of PA hypoxic contraction (Fig. 4 c-d), in a manner similar to that elicited by antioxidants ${ }^{36}$. These observations suggest a physiological role for NCLX in hypoxic pulmonary vasoconstriction (HPV), a phenomenon necessary to match lung ventilation with perfusion, which is crucial for cardiovascular homeostasis. 


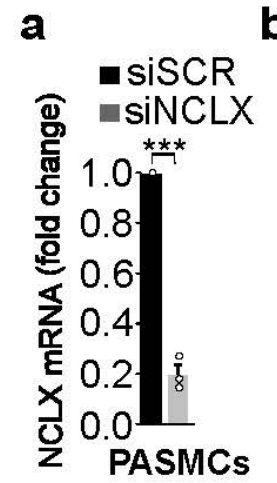

b $\widehat{3} 30$
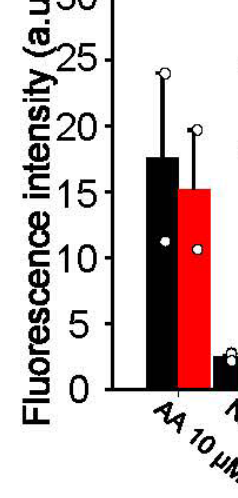

$\stackrel{\text { n.s. }}{* * * *}$

*** Superoxide (PASMCs)
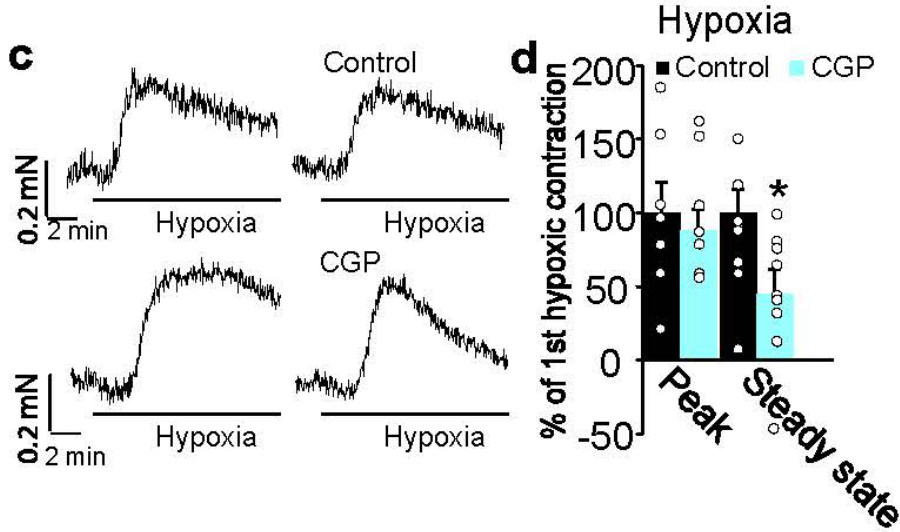

Fig. 4. $\mathrm{Na}^{+}$mito influx inhibition abolishes hypoxic pulmonary vasoconstriction. a, Effectiveness of NCLX silencing in mice pulmonary artery smooth muscle cells (PASMCs) measured by quantitative RT-PCR analysis, $n=3$. b, Superoxide detection by fluorescence microscopy after incubation with DHE in PASMCs treated with siSCR or siNCLX in normoxia $(\mathrm{Nx})$ or hypoxia $(\mathrm{Hp}$; $\left.1 \% \mathrm{O}_{2}\right), \mathrm{n}=3$. c-d, Representative traces (c) and average values (d) of HPV measured in rat pulmonary arteries. Each artery was exposed twice to hypoxia, and the second hypoxic challenge was performed in the absence or the presence of $30 \mu \mathrm{M} \quad$ CGP-37157, $\mathrm{n}=7-9$. Student's t-test for pairwise comparisons and one-way ANOVA with Tukey's test for multiple comparisons: $* \mathrm{p}<0.05$, $* *<\mathrm{p} 0.01, \quad * * * \quad \mathrm{p}<0.001 . \quad \mathbf{b}$, statistical comparisons shown only for $\mathrm{Nx}$ vs 0-10 groups.

The role of $\mathrm{Na}^{+}$in the cell has been restricted to the maintenance of membrane potentials and to aid ion exchange at membranes $\left(\mathrm{Na}^{+} / \mathrm{K}^{+}, \mathrm{Na}^{+} / \mathrm{Ca}^{2+}\right.$, etc.). Here we show that $\mathrm{Na}^{+}$directly modulates mitochondrial OXPHOS and ROS signalling with significant implications in tissue homeostasis. In addition, we identify mitochondrial calcium phosphate precipitates as a significant source of mitochondrial soluble $\mathrm{Ca}^{2+}$ in physiological conditions.

Interestingly, we noticed that $\mathrm{Na}^{+}$only exerted its effect on CII+CIII or G3PDH+CIII activities, but not on CI+CIII activity. The only model that may explain this selective behaviour is the assembly of the respiratory complexes into supercomplexes. Thus, $\mathrm{CoQ}_{10}$ transfer from $\mathrm{CI}$ to CIII does not decrease in the presence of $\mathrm{Na}^{+}$because CI+CIII supercomplex activity does not depend on membrane fluidity, as it may utilize the $\mathrm{CoQ}\left(\mathrm{CoQ}{ }^{\mathrm{NADH}}\right)$ partially trapped in the supercomplex microenvironment ${ }^{37,38}$. This is supported by a recent report describing the structure of the $\mathrm{I}+\mathrm{III}_{2}$ supercomplex ${ }^{39}$. On the other hand, CoQ transfer from either CII or G3PD to CIII 
strongly relies on membrane fluidity and consequently, both CII+CIII and G3PD+III (i.e. $\mathrm{CoQ}^{\mathrm{FAD}}$ ) activities are lower in the presence of $\mathrm{Na}^{+}$. Therefore, the partial segmentation of the CoQ pool into $\mathrm{CoQ}{ }^{\mathrm{NADH}}$ and $\mathrm{CoQ}^{\mathrm{FAD}}$ allows the specific CIII-dependent ROS generation upon $\mathrm{Na}^{+}: \mathrm{PC}$ interaction in response to acute hypoxia meanwhile respiration can be maintained by means of supercomplex function.

A recent report described the beneficial role of NCLX by decreasing ROS production in a murine model of cardiac ischemia-reperfusion ${ }^{12}$. Although it could seem contradictory with the findings reported here, both roles of NCLX are compatible. During cardiac ischemia succinate accumulates, which causes an elevation of the $\mathrm{CoQH}_{2} / \mathrm{CoQ}$ ratio and, at reperfusion induces superoxide production by reverse electron transport (RET) through $\mathrm{CI}^{4}$. There are three ways by which NCLX activation could hinder ROS production by RET during reperfusion: a) $\mathrm{Na}^{+}$:phospholipid interaction would restrain $\mathrm{CoQH}_{2}$ diffusion towards $\mathrm{CI}$ through the reduction of the IMM fluidity; b) $\mathrm{CoQH}_{2} / \mathrm{CoQ}$ ratio drops during hypoxia in a NCLX-dependent manner, what would decrease RET during reoxygenation; c) $\mathrm{Ca}^{2+}$ extrusion by NCLX would prevent further TCA-cycle dehydrogenases activation, limiting the production of reducing equivalents to OXPHOS complexes. Thus, our model fully supports the role of NCLX in attenuating ROS production and injury upon cardiac reperfusion ${ }^{12}$. Indeed, the present mechanism may represent a way by which mitochondria reduce potentially toxic CI RET-derived ROS and induce an adaptive short-time elevation of CIII-dependent ROS production.

In summary, our findings open new horizons for the study of mitochondrial OXPHOS and signalling. The impact of $\mathrm{Na}^{+}$as a second messenger on mitochondrial homeostasis had been ignored, but our results suggest it likely plays a pivotal role in mitochondrial physiology in health and disease. 


\section{References}

1 Sena, L. A. \& Chandel, N. S. Physiological roles of mitochondrial reactive oxygen species. Mol Cell 48, 158-167, (2012).

2 Shadel, G. S. \& Horvath, T. L. Mitochondrial ROS signaling in organismal homeostasis. Cell 163, 560-569, (2015).

3 Dan Dunn, J., Alvarez, L. A., Zhang, X. \& Soldati, T. Reactive oxygen species and mitochondria: A nexus of cellular homeostasis. Redox Biol 6, 472-485, (2015).

4 Chouchani, E. T. et al. Ischaemic accumulation of succinate controls reperfusion injury through mitochondrial ROS. Nature 515, 431-435, (2014).

5 Guzy, R. D. \& Schumacker, P. T. Oxygen sensing by mitochondria at complex III: the paradox of increased reactive oxygen species during hypoxia. Exp Physiol 91, 807-819, (2006).

6 Fernández-Agüera, M. C. et al. Oxygen Sensing by Arterial Chemoreceptors Depends on Mitochondrial Complex I Signaling. Cell Metab 22, 825-837, (2015).

7 Hernansanz-Agustín, P. et al. Acute hypoxia produces a superoxide burst in cells. Free Radic Biol Med 71, 146-156, (2014).

8 Sylvester, J. T., Shimoda, L. A., Aaronson, P. I. \& Ward, J. P. Hypoxic pulmonary vasoconstriction. Physiol Rev 92, 367-520, (2012).

9 Giorgi, C., Marchi, S. \& Pinton, P. The machineries, regulation and cellular functions of mitochondrial calcium. Nat Rev Mol Cell Biol 19, 713-730, (2018).

10 Bers, D. M., Barry, W. H. \& Despa, S. Intracellular Na+ regulation in cardiac myocytes. Cardiovasc Res 57, 897-912, (2003).

11 Hernansanz-Agustín, P. et al. Mitochondrial complex I deactivation is related to superoxide production in acute hypoxia. Redox Biol 12, 1040-1051, (2017).

12 Luongo, $\mathrm{T}$. S. et al. The mitochondrial $\mathrm{Na}+/ \mathrm{Ca} 2+$ exchanger is essential for $\mathrm{Ca} 2+$ homeostasis and viability. Nature 545, 93-97, (2017).

13 Hamanaka, R. B. \& Chandel, N. S. Mitochondrial reactive oxygen species regulate cellular signaling and dictate biological outcomes. Trends Biochem Sci 35, 505-513, (2010).

14 Babot, M., Birch, A., Labarbuta, P. \& Galkin, A. Characterisation of the active/de-active transition of mitochondrial complex I. Biochim Biophys Acta 1837, 1083-1092, (2014).

15 Zhu, J., Vinothkumar, K. R. \& Hirst, J. Structure of mammalian respiratory complex I. Nature 536, 354-358, (2016).

16 Fiedorczuk, K. et al. Atomic structure of the entire mammalian mitochondrial complex I. Nature 538, 406-410, (2016).

17 Greenawalt, J. W., Rossi, C. S. \& Lehninger, A. L. Effect of Active Accumulation of Calcium and Phosphate Ions on the Structure of Rat Liver Mitochondria. J Cell Biol 23, 21-38, (1964).

18 Wolf, S. G. et al. 3D visualization of mitochondrial solid-phase calcium stores in whole cells. Elife 6, (2017). 
19 Chandel, N. S. et al. Reactive oxygen species generated at mitochondrial complex III stabilize hypoxia-inducible factor-1alpha during hypoxia: a mechanism of $\mathrm{O} 2$ sensing. $\mathrm{J}$ Biol Chem 275, 25130-25138, (2000).

20 Guzy, R. D. et al. Mitochondrial complex III is required for hypoxia-induced ROS production and cellular oxygen sensing. Cell Metab 1, 401-408, (2005).

21 Carafoli, E., Tiozzo, R., Lugli, G., Crovetti, F. \& Kratzing, C. The release of calcium from heart mitochondria by sodium. J Mol Cell Cardiol 6, 361-371, (1974).

22 Cox, D. A. \& Matlib, M. A. A role for the mitochondrial $\mathrm{Na}(+)-\mathrm{Ca} 2+$ exchanger in the regulation of oxidative phosphorylation in isolated heart mitochondria. J Biol Chem 268, 938-947, (1993).

23 Acín-Pérez, R., Fernández-Silva, P., Peleato, M. L., Pérez-Martos, A. \& Enríquez, J. A. Respiratory active mitochondrial supercomplexes. Mol Cell 32, 529-539, (2008).

24 Lenaz, G. \& Genova, M. L. Mobility and function of coenzyme Q (ubiquinone) in the mitochondrial respiratory chain. Biochim Biophys Acta 1787, 563-573, (2009).

25 Budin, I. et al. Viscous control of cellular respiration by membrane lipid composition. Science 362, 1186-1189, (2018).

26 Petit, J. M., Maftah, A., Ratinaud, M. H. \& Julien, R. 10N-nonyl acridine orange interacts with cardiolipin and allows the quantification of this phospholipid in isolated mitochondria. Eur J Biochem 209, 267-273, (1992).

27 Gallet, P. F., Petit, J. M., Maftah, A., Zachowski, A. \& Julien, R. Asymmetrical distribution of cardiolipin in yeast inner mitochondrial membrane triggered by carbon catabolite repression. Biochem J 324 ( Pt 2), 627-634, (1997).

28 Horvath, S. E. \& Daum, G. Lipids of mitochondria. Prog Lipid Res 52, 590-614, (2013).

29 Sarewicz, M. \& Osyczka, A. Electronic connection between the quinone and cytochrome $\mathrm{C}$ redox pools and its role in regulation of mitochondrial electron transport and redox signaling. Physiol Rev 95, 219-243, (2015).

30 Pabst, G. et al. Rigidification of neutral lipid bilayers in the presence of salts. Biophys J 93, 2688-2696, (2007).

31 Böckmann, R. A., Hac, A., Heimburg, T. \& Grubmüller, H. Effect of sodium chloride on a lipid bilayer. Biophys J 85, 1647-1655, (2003).

32 Cordomí, A., Edholm, O. \& Perez, J. J. Effect of ions on a dipalmitoyl phosphatidylcholine bilayer. a molecular dynamics simulation study. J Phys Chem B 112, 1397-1408, (2008).

33 Michelakis, E. D., Thebaud, B., Weir, E. K. \& Archer, S. L. Hypoxic pulmonary vasoconstriction: redox regulation of $\mathrm{O} 2$-sensitive $\mathrm{K}+$ channels by a mitochondrial $\mathrm{O} 2$ sensor in resistance artery smooth muscle cells. J Mol Cell Cardiol 37, 1119-1136, (2004).

34 Moreno, L. et al. Ceramide mediates acute oxygen sensing in vascular tissues. Antioxid Redox Signal 20, 1-14, (2014).

35 Desireddi, J. R., Farrow, K. N., Marks, J. D., Waypa, G. B. \& Schumacker, P. T. Hypoxia increases ROS signaling and cytosolic $\mathrm{Ca}(2+)$ in pulmonary artery smooth muscle cells of mouse lungs slices. Antioxid Redox Signal 12, 595-602, (2010). 
36 Connolly, M. J., Prieto-Lloret, J., Becker, S., Ward, J. P. \& Aaronson, P. I. Hypoxic pulmonary vasoconstriction in the absence of pretone: essential role for intracellular $\mathrm{Ca} 2+$ release. J Physiol 591, 4473-4498, (2013).

37 Lapuente-Brun, E. et al. Supercomplex assembly determines electron flux in the mitochondrial electron transport chain. Science 340, 1567-1570, (2013).

38 Enríquez, J. A. Supramolecular Organization of Respiratory Complexes. Annu Rev Physiol 78, 533-561, (2016).

39 Letts, J. A., Fiedorczuk, K., Degliesposti, G., Skehel, M. \& Sazanov, L. A. Structures of Respiratory Supercomplex I+III2 Reveal Functional and Conformational Crosstalk. Mol Cell, (2019).

40 Navarro-Antolín, J., Rey-Campos, J. \& Lamas, S. Transcriptional induction of endothelial nitric oxide gene by cyclosporine A. A role for activator protein-1. J Biol Chem 275, 3075-3080, (2000).

41 Muñoz, C. et al. Transcriptional up-regulation of intracellular adhesion molecule-1 in human endothelial cells by the antioxidant pyrrolidine dithiocarbamate involves the activation of activating protein-1. J Immunol 157, 3587-3597, (1996).

42 McCombs, J. E. \& Palmer, A. E. Measuring calcium dynamics in living cells with genetically encodable calcium indicators. Methods 46, 152-159, (2008).

43 Cogliati, S. et al. Mechanism of super-assembly of respiratory complexes III and IV. Nature 539, 579-582, (2016).

44 Schägger, H. Tricine-SDS-PAGE. Nat Protoc 1, 16-22, (2006).

45 Zhang, H. Thin-Film Hydration Followed by Extrusion Method for Liposome Preparation. Methods Mol Biol 1522, 17-22, (2017).

46 Rouser, G., Fkeischer, S. \& Yamamoto, A. Two dimensional then layer chromatographic separation of polar lipids and determination of phospholipids by phosphorus analysis of spots. Lipids 5, 494-496, (1970).

47 Scorrano, L. et al. A distinct pathway remodels mitochondrial cristae and mobilizes cytochrome c during apoptosis. Dev Cell 2, 55-67, (2002).

48 Scialo, F. et al. Mitochondrial ROS Produced via Reverse Electron Transport Extend Animal Lifespan. Cell Metab 23, 725-734, (2016).

49 Rodríguez-Aguilera, J. C., Cortés, A. B., Fernández-Ayala, D. J. \& Navas, P. Biochemical Assessment of Coenzyme Q10 Deficiency. J Clin Med 6, (2017).

50 Yubero, D. et al. Secondary coenzyme Q10 deficiencies in oxidative phosphorylation (OXPHOS) and non-OXPHOS disorders. Mitochondrion 30, 51-58, (2016).

51 Stone, T. J., Buckman, T., Nordio, P. L. \& McConnell, H. M. Spin-labeled biomolecules. Proc Natl Acad Sci U S A 54, 1010-1017, (1965).

52 Martínez-Ruiz, A. et al. RNase U2 and alpha-sarcin: a study of relationships. Methods Enzymol 341, 335-351, (2001).

53 Gasset, M., Martínez del Pozo, A., Oñaderra, M. \& Gavilanes, J. G. Study of the interaction between the antitumour protein alpha-sarcin and phospholipid vesicles. Biochem J 258, 569-575, (1989). 


\section{Methods}

\section{Animals, cell culture and transfection}

All animal experiments were performed following the Guide for the Care and Use of Laboratory Animals and were approved by the institutional ethics committee of the Universidad Autónoma de Madrid or the Universidad Complutense de Madrid, Spain, in accordance with the European Union Directive of 22 September 2010 (2010/63/UE) and with the Spanish Royal Decree of 1 February 2013 (53/2013). All efforts were made to minimize the number of animals used and their suffering.

Cells were routinely maintained in cell culture incubators ( $95 \%$ air, $5 \% \mathrm{CO}_{2}$ in gas phase, $37^{\circ} \mathrm{C}$ ). Bovine aortic endothelial cells (BAECs) were isolated as described ${ }^{40}$ and cultured in RPMI 1640 supplemented with $15 \%$ heat-inactivated fetal bovine serum (FBS), $100 \mathrm{U} / \mathrm{mL}$ penicillin and 100 $\mu \mathrm{g} / \mathrm{mL}$ streptomycin. Mouse embryonic fibroblasts (MEFs) were isolated as described previously ${ }^{12}$ and cultured in DMEM supplemented with $10 \%$ heat-inactivated fetal bovine serum (FBS), $100 \mathrm{U} / \mathrm{mL}$ penicillin and $100 \mu \mathrm{g} / \mathrm{mL}$ streptomycin. Human umbilical vein endothelial cells (HUVECs) were isolated as described ${ }^{41}$ and cultured in Medium 199 supplemented with $20 \%$ heat-inactivated FBS, $16 \mathrm{U} / \mathrm{mL}$ heparin, $100 \mathrm{mg} / \mathrm{L}$ ECGF (endothelial cell growth factor), $20 \mathrm{mM}$ HEPES (4-(2-hydroxyethyl)piperazine-1-ethanesulfonic acid), $100 \mathrm{U} / \mathrm{mL}$ penicillin and 100 $\mu \mathrm{g} / \mathrm{mL}$ streptomycin. BAECs were used between passages 3 and 9 and HUVECs between passages 3 and 7. Endothelial morphology was assessed by visual inspection.

Rat pulmonary artery smooth muscle cells (PASMCs) were isolated as described ${ }^{34}$ and cultured in Dulbecco's modified Eagle's medium (DMEM) supplemented with $10 \%$ FBS, $1.1 \mathrm{~g} / \mathrm{L}$ pyruvate, $1 \%$ non-essential amino acids, $100 \mu \mathrm{g} / \mathrm{mL}$ streptomycin and $100 \mathrm{U} / \mathrm{mL}$ penicillin. PASMCs were used between passages 2 and 3 .

Hepatoma cell line HepG2 were cultured at $37{ }^{\circ} \mathrm{C}$ in DMEM supplemented with $10 \%$ heatinactivated FBS, $20 \mathrm{mM}$ HEPES, $100 \mathrm{U} / \mathrm{mL}$ penicillin and $100 \mu \mathrm{g} / \mathrm{mL}$ streptomycin.

Transfection of $30 \mathrm{nM}$ siRNA or $0.25 \mu \mathrm{g}$ Cyto-GEM-GECO, Cepia $2 \mathrm{mt}$, human NCLX (pNCLX; Addgene), S468T NCLX (dnNCLX), C199S pHyPer-Myto (mitosypHer), pHyPer-cyto (cytohyper) or pDsRed2-Mito (mitoRFP) vector DNA per $0.8 \mathrm{~cm}^{2}$ well was carried out using Lipofectamine 2000 (Invitrogen). Acute ablation in NCLX flx/flx MEFs was obtained by infection with adenoviruses expressing cytomegalovirus (CMV)-Cre (ad-CRE; $300 \mathrm{pfu} / \mathrm{cell}$; Vector Biolabs). In PASMCs, $10 \mathrm{nM}$ siRNA was transfected using Lipofectamine RNA iMAX (Invitrogen); in parallel experiments, siRNA efficiency was measured by RT-PCR using commercially available primers (TaqMan Gene Expression Assay Rn01481405_m1, Applied Biosystems). Experiments were carried out 48 to $72 \mathrm{~h}$ after transfection.

Immortalization of HUVECs and MEFs was performed by retrovirus infection. 293T cells were transfected with 12 ug pCL-Ampho and 12 ug pBABE-SV40-puro using Lipofectamine RNA iMAX (Invitrogen). Two days later, 1/2 dilution of 293T cells media and $8 \mathrm{ug} / \mathrm{mL}$ of polybrene were added on MEFs or HUVECs cultures during 4 hours. Then, the infection was repeated, but adding $4 \mathrm{ug} / \mathrm{mL}$ of polybrene overnight. Next day, the first infection was repeated and, upon 4 hours, MEFs or HUVECs were passaged with 1/1000 of puromicin.

\section{SiRNA preparation}

Four double-stranded siRNA against bovine NCLX were designed and purchased from Integrated DNA Technologies and Dharmacon (sense sequences of siNCLX1: AGCGGCCACUCAACUGCCU; SiNCLX2: GUUUGGAACUGAAACACU and UUCCGUAAGUGUUUCAGU mixed; siNCLX3: AAAGGUGGAAGUAAUCAC and ACGUAUUGUGAUUACUUC mixed; siNCLX4: GAAUUUGGAGUGAUUCAC and 
UUUUCAAGUGAAUCACUC mixed). Double-stranded siRNAs against bovine NDUFS4 and RISP were designed and purchased from Integrated DNA Technologies (NDUFS4 sense sequence GCUGCCGUUUCCGUUUCCAAGGUUUTT; NDUFS2 sense sequence TCGGACAGTCGACATTGGGATT; RISP sense sequence CCAAGAAUGUCGUCUCUCAGUUUTT). Scrambled siRNA (siSCR) was purchased from Santa Cruz Biotechnology.

siRNA against rat NCLX (SL8B1 gene) was purchased from OriGene (SLC8B1 Trilencer-27 Rat siRNA).

\section{Detection of superoxide by fluorescence microscopy in fixed cells}

Cells were seeded on glass coverslips one day before experimentation. In some experiments, 1 $\mu \mathrm{M}$ rotenone or $10 \mu \mathrm{M}$ 7-chloro-5-(2-chlorophenyl)-1,5-dihydro-4,1-benzothiazepin-2(3H)-one (CGP-37157) was added $30 \mathrm{~min}$ before experimentation and maintained during the experiment. For treatments in hypoxia, all the solutions were pre-equilibrated in hypoxic conditions before use; plated cells were introduced into an Invivo2 400 workstation (Ruskinn) set at $1 \% \mathrm{O}_{2}, 5 \%$ $\mathrm{CO}_{2}, 37^{\circ} \mathrm{C}$, and incubated for the indicated times $(0,15,30,45$ and $60 \mathrm{~min})$ in fresh medium, washed three times with Hank's Balanced Salt Solution with $\mathrm{Ca}^{2+} / \mathrm{Mg}^{2+}(\mathrm{HBSS}+\mathrm{Ca} / \mathrm{Mg}$, which contains $141 \mathrm{mM} \mathrm{Na}{ }^{+}$and $5.8 \mathrm{mM} \mathrm{K}^{+}$) and incubated with $5 \mu \mathrm{M}$ dihydroethidium (DHE) in $\mathrm{HBSS}+\mathrm{Ca} / \mathrm{Mg}$ for $10 \mathrm{~min}$ in the dark. Excess probe was removed by three washes with $\mathrm{HBSS}+\mathrm{Ca} / \mathrm{Mg}$, cells were fixed with $4 \%$ paraformaldehyde (PFA), and incubated in the dark at 4 ${ }^{\circ} \mathrm{C}$ for $15 \mathrm{~min}$. After fixation, the cells were again washed three times with $\mathrm{HBSS}+\mathrm{Ca} / \mathrm{Mg}$ and coverslips were placed on slides. For normoxic treatments, the medium was changed to fresh normoxic medium, and cells were treated as for hypoxic cells but in a standard cell incubator. Images (three images per each coverslip; the number of independent experiments is described in the figure legends) were taken with a Leica DMR fluorescence microscope with a $63 \times$ objective, using the 546-12/560 excitation/emission filter pairs, and quantified using ImageJ software $(\mathrm{NIH})$. The same threshold was set for all the images and the mean value from histograms was averaged for the three images of each coverslip.

\section{Detection of intracellular calcium, sodium, ROS and mitochondrial membrane potential by live imaging fluorescence microscopy}

Cells were seeded in 6-well plates one day before experimentation. Plated cells were washed three times with $\mathrm{HBSS}+\mathrm{Ca} / \mathrm{Mg} \pm$ Glucose and incubated with $30 \mathrm{nM}$ tetramethylrhodamine methyl ester (TMRM), $1 \mu \mathrm{M}$ Fluo-4 AM, $10 \mu \mathrm{M}$ CoroNa Green AM or $10 \mu \mathrm{M}$ 6-carboxy-2',7'dichlorodihydrofluorescein diacetate (CDCFDA) for $20 \mathrm{~min}$ at $37^{\circ} \mathrm{C}$ in the dark. CDCFDA, CoroNa Green AM and Fluo-4 AM were then washed out and new $\mathrm{HBSS}+\mathrm{Ca} / \mathrm{Mg}$ was added. In some experiments, $10 \mu \mathrm{M}$ CGP-37157 or $10 \mu \mathrm{M}$ dimethylmalonate (DMM) were also added and maintained during the remainder of the experiment. For Fluo-4 AM imaging, cells were further incubated for $30 \mathrm{~min}$ at $37^{\circ} \mathrm{C}$ in the dark to allow complete de-esterification of the probe. After this time, the plate was placed into a Leica DM 16000B fluorescence microscope equipped with a Leica DFC360FXcamera, an automated stage for live imaging and a thermostated hypoxic cabinet. The planes were focused for image capture, and images were taken with a $20 \times$ objective every 2 min during $40 \mathrm{~min}$, providing a total of 21 cycles. Normoxia experiments started and ended at $20 \% \mathrm{O}_{2}$ and $5 \% \mathrm{CO}_{2}$, whereas hypoxia experiments started at $20 \% \mathrm{O}_{2}$ and $5 \% \mathrm{CO}_{2}$ and then were switched to $2 \% \mathrm{O}_{2}$ and $5 \% \mathrm{CO}_{2}$ in cycle 2 . The excitation/emission filter pairs used were as follows: and TMRM, and 480-40/505 for CDCFDA, Fluo-4 AM and CoroNa Green AM. Images were quantified with Leica Las-AF software. Three independent experiments were performed for each condition. For each experiment and condition, four regions of interest (ROIs) were created, each ROI surrounding an individual cell, and the mean fluorescence of each ROI 
for each time cycle was collected. In some analyses, for each experiment and condition, four identical linear ROIs were created and the maximum peak value of cycles $0,5,10,15$ and 20 were collected for each ROI.

\section{Detection of cytosolic calcium, sodium and $\mathrm{H}_{2} \mathrm{O}_{2}$, and intramitochondrial $\mathrm{pH}, \mathrm{Ca}^{2+}$ and $\mathrm{Na}^{+}$by live imaging confocal microscopy}

To detect intracellular calcium and sodium, cells were seeded one day before experimentation, washed three times with $\mathrm{HBSS}+\mathrm{Ca} / \mathrm{Mg}+\mathrm{Glucose}$ and in some experiments incubated with $1 \mu \mathrm{M}$ Fluo-4 AM, Corona Red or $5 \mu \mathrm{M}$ CoroNa Green AM, Asante Natrium 2 AM (ANG2 AM) for 30 min at $37^{\circ} \mathrm{C}$ in the dark. In some experiments, $10 \mu \mathrm{M}$ CGP-37157, $10 \mu \mathrm{M}$ DMM, $1 \mu \mathrm{M}$ FCCP, 1 $\mu \mathrm{M}$ rotenone, or $1 \mu \mathrm{M}$ Ru360 were also added and maintained during the remainder of the experiment. $100 \mu \mathrm{M}$ Histamine was added acutely in control experiments. CoroNa Green AM, ANG2 AM, Corona Red and Fluo-4 AM were then washed out and new HBSS+Ca/Mg plus 25 $\mathrm{mM}$ glucose was added. For Fluo-4 AM and ANG2 AM imaging, cells were further incubated for $30 \mathrm{~min}$ at $37^{\circ} \mathrm{C}$ in the dark to allow complete de-esterification of the probe. After this time, the plate was placed into a Leica SP-5 confocal microscope, an automated stage for live imaging and a thermostated hypoxic cabinet. The planes were focused for image capture and images were taken with a $63 \times$ objective. In some experiments images were taken every 2 min during $40 \mathrm{~min}$, providing a total of 21 cycles and in others every five minutes. Normoxia experiments started and ended at $20 \% \mathrm{O}_{2}$ and $5 \% \mathrm{CO}_{2}$, whereas hypoxia experiments started at $20 \% \mathrm{O}_{2}$ and $5 \% \mathrm{CO}_{2}$ and then were switched to $1 \% \mathrm{O}_{2}$ and $5 \% \mathrm{CO}_{2}$ in cycle 1 . In other experiments hypoxia conditions were set at the middle of the protocol, allowing the quantification of the same cells during normoxia and hypoxia. Loaded cells were excited with an argon/krypton laser using the $496 \mathrm{~nm}$ line, except Corona Red which was excited with the 514 line. Fluorescence emission of Fluo-4 AM, ANG2 AM and CoroNa Green was detected in 515-575 nm range, and 555-575 nm for Corona Red.

To detect intramitochondrial $\mathrm{pH}$, cells were transfected with the ratiometric probe mitosypHer in 8 -well plates the day before the experiment. The same protocol as above was used, except that the objective was $63 \times$ and imaging time was $30 \mathrm{~min}$, with 7 cycles of $5 \mathrm{~min}$. Excitation was performed with a 405 diode laser for $405 \mathrm{~nm}$ line and an argon/krypton laser for $488 \mathrm{~nm}$ line, and fluorescent emission was recorded at the 515-535 nm range.

For $\mathrm{H}_{2} \mathrm{O}_{2}$ detection, cells were transfected with the non-targeted version of HyPer (pHyPer-cyto) following the same procedure for live imaging as with mitosypHer.

Images were quantified with ImageJ software. Three or four independent experiments were performed for each condition. For each experiment and condition in loaded cells, four identical linear regions of interest (ROIs) were quantified, and for each time point the mean of these ROIs was obtained.

For cytosolic $\mathrm{Ca}^{2+}$ detection, cells were transfected with the non-targeted version of GEM-GECO (cyto-GEM-GECO) in 8-well plates the day before the experiment. The objective was $63 \times$ and imaging time was $45 \mathrm{~min}$, with 9 cycles of $5 \mathrm{~min}$. Excitation was performed with a 405-diode laser for $405 \mathrm{~nm}$ line, and fluorescent emission was recorded at 460 and $510 \mathrm{~nm}$ lines.

For mitochondrial $\mathrm{Ca}^{2+}$ detection, cells were transfected with the mitochondrial-targeted Cepia2mt or mito-GEM-GECO in 8-well plates the day before the experiment. The objective was $63 \times$ and imaging time was $40 \mathrm{~min}$, with 20 cycles of $2 \mathrm{~min}$. Samples transfected with Cepia2mt were excited with an argon/krypton laser using the $488 \mathrm{~nm}$ line and fluorescence emission was detected in the 515-535 $\mathrm{nm}$ range.

For mitochondrial $\mathrm{Na}^{+}$detection, cells were washed three times with $\mathrm{HBSS}+\mathrm{Ca} / \mathrm{Mg}+\mathrm{Glucose}$, incubated for 1 hour with $10 \mu \mathrm{M}$ CoroNa Green AM, washed three times again with $\mathrm{HBSS}+\mathrm{Ca} / \mathrm{Mg}+$ Glucose and incubated a further hour in $\mathrm{HBSS}+\mathrm{Ca} / \mathrm{Mg}+\mathrm{Glucose}$. During this period Corona Green is actively pumped out from the cytosol. Then, after washing three more 
times with $\mathrm{HBSS}+\mathrm{Ca} / \mathrm{Mg}+\mathrm{Glucose}$, cells were placed in the microscope. The objective was $63 \times$ and imaging time was $25 \mathrm{~min}$, with 15 cycles of $2.5 \mathrm{~min}$. Samples were excited with an argon/krypton laser using the $488 \mathrm{~nm}$ line and fluorescence emission was detected in the 500-575 nm range.

Calibrations in $\mathrm{Ca}^{2+}$ measurements were performed as previously described ${ }^{42}$.

\section{Western blot analysis}

Protein samples were extracted with non-reducing Laemmli buffer without bromophenol blue and quantified by Bradford assay. Extracts were loaded onto $10 \%$ standard polyacrylamide gel electrophoresis after adding 5\% 2-mercaptoethanol, and subsequently transferred to nitrocellulose membranes or PVDF membranes. The following antibodies were used: polyclonal anti-NCLX antibodies (ab136975, Abcam; ARP44042_P050, Aviva Systems Biology), monoclonal anti-Fp70 (459200; Invitrogen), monoclonal anti-NDUFS4 antibody (ab87399; Abcam), monoclonal antiRISP (UQCRFS1) antibody (ab14746; Abcam), and monoclonal anti- $\alpha$-tubulin antibody (\#T6199, Sigma). Antibody binding was detected by chemiluminescence with species-specific secondary antibodies labelled with horseradish peroxidase (HRP), and visualized on a digital luminescent image analyser (Fujifilm LAS-4000), with the exception of ab136975 and 459200 which were detected by fluorescence as previously described ${ }^{43}$.

\section{Quantitative Real Time PCR}

Total RNA was extracted from PASMCs using Trizol reagent (Vitro) and $0.5 \mu \mathrm{g}$ was reversetranscribed (Gene Amp Gold RNA PCR Core Kit; Applied Biosystems). PCR was performed with GotaqqPCR Master Mix (Promega) with $1 \mu \mathrm{L}$ of cDNA and specific primer pairs (Rn01481405_m1). $\beta$-actin mRNA was measured as an internal sample control.

\section{Measurement of cellular oxygen consumption}

Oxygen consumption rate (OCR) was measured using an XF24 Extracellular Flux Analyzer (Seahorse Bioscience, North Billerica, MA, USA). BAECs, $6 \times 10^{4}$ per well (6-7 wells per treatment for each independent experiment) were plated one day before the experiment. Cells were preincubated with unbuffered DMEM supplemented with $25 \mathrm{mM}$ glucose, $1 \mathrm{mM}$ pyruvate, and $2 \mathrm{mM}$ glutamine for $1 \mathrm{~h}$ at $37^{\circ} \mathrm{C}$ in an incubator without $\mathrm{CO}_{2}$ regulation. OCR measurements were programmed with successive injections of unbuffered DMEM, $5 \mu \mathrm{g} / \mathrm{mL}$ oligomycin, 300 $\mathrm{nM}$ carbonyl cyanide 4-(trifluoromethoxy) phenylhydrazone (FCCP), and $1 \mu \mathrm{M}$ rotenone plus 1 $\mu \mathrm{M}$ antimycin A. DMSO or $10 \mu \mathrm{M}$ CGP-37157 were added before starting the measurements. In experiments using silenced cells, as proliferation and cell growth may vary after plating, protein concentration was quantified by the BCA assay to normalize the OCR. Calculations were performed following the manufacturer's instructions. After measuring basal respiration, oligomycin was added to inhibit respiration (by blocking $\mathrm{H}^{+}$-ATPase); therefore, the amount of oxygen used to produce ATP by OXPHOS is estimated from the difference with basal oxygen consumption (i.e., coupling efficiency). FCCP uncouples OXPHOS by translocating $\mathrm{H}^{+}$from intermembrane space to matrix, thus maximizing electron flux through the electron transport chain (ETC), giving the maximal respiration rate. This treatment provides information about the stored energy in mitochondria that a cell could use in an energetic crisis (i.e., reserve capacity). Antimycin A and rotenone block complexes III and I, respectively, consequently inhibiting electron flux through the ETC and eliminating any $\mathrm{H}^{+}$translocation, therefore, the leftover value is non-mitochondrial respiration, that is, the oxygen consumed by other enzymes in the cell.

\section{Fluorescent labelling of ND3 Cys-39 from isolated mitochondrial membranes}

SMP were prepared as previously described ${ }^{11}$. SMP protein amount was determined by BCA assay and then proteins were solubilized with $4 \mathrm{~g} / \mathrm{g}$ digitonin, incubated $5 \mathrm{~min}$ on ice and 
centrifuged for $30 \mathrm{~min}$ at $16,000 \mathrm{~g}, 4{ }^{\circ} \mathrm{C}$. Samples were split into two parts: one part was incubated at $37{ }^{\circ} \mathrm{C}$ for 60 min to fully deactivate complex I and the other part was kept on ice. Samples were then incubated with Bodipy-TMR C5-maleimide (Invitrogen) for $20 \mathrm{~min}$ at $15{ }^{\circ} \mathrm{C}$ in the dark; then, $1 \mathrm{mM}$ cysteine was added and the samples were further incubated for 5 min. After this time, the samples were precipitated twice with acetone, centrifuged at 9,500 $\mathrm{g}$ for 10 min at $4{ }^{\circ} \mathrm{C}$ in the dark, and the resulting pellet was resuspended in non-reducing Laemmli loading buffer. For each sample, $100 \mu \mathrm{g}$ were loaded onto 10\% Tricine-SDS-PAGE gels as described $^{44}$. Total protein staining was performed with Sypro Ruby (Invitrogen). The images of the different fluorophores were obtained using a digital fluorescent image analyzer (Fujifilm LAS-4000). Images were quantified using ImageQuant TL7.0 software.

\section{Mitochondria isolation and measurement of $\mathrm{H}_{2} \mathrm{O}_{2}$ and oxygen consumption}

Mitochondria were isolated from BAECs with a protocol adapted for cell culture ${ }^{37}$. Briefly, after resuspending BAECs with a sucrose buffer in a glass Elvehjem potter, homogenization was performed by up and down strokes using a motor-driven Teflon pestle. Successive homogenization-centrifugation steps yielded the mitochondria-containing fraction.

$\mathrm{H}_{2} \mathrm{O}_{2}$ production from isolated rat heart mitochondria was performed in an O2k Oxygraph instrument (Oroboros Instruments). $500 \mu \mathrm{g}$ of isolated rat heart mitochondria were loaded in $\mathrm{KCl}$ buffer with Amplex Red: $150 \mathrm{mM} \mathrm{KCl}, 10 \mathrm{mM} \mathrm{K}_{2} \mathrm{HPO}_{4}, 1 \mathrm{mM}$ EDTA, $5 \mathrm{mM} \mathrm{MgCl} 2,1 \mathrm{mg} / \mathrm{mL}$ BSA, $15 \mu \mathrm{g} / \mathrm{ml}$ Horseradish peroxidase (HRP), $15 \mu \mathrm{g} / \mathrm{ml}$ superoxide dismutase (SOD) and $25 \mu \mathrm{M}$ Amplex Red. Recordings started before the addition of substrates: $5 \mathrm{mM}$ glutamate and $5 \mathrm{mM}$ malate (GM); and the indicated amounts of $\mathrm{NaCl}$ and/or $\mathrm{CaCl}_{2}$ were subsequently added.

Oxygen consumption was determined with an oxytherm Clark-type electrode (Hansatech) as described $^{37}$. Briefly, isolated mitochondria from BAECs $(150 \mu \mathrm{g})$, MEFs WT or MEFs KO were resuspended in MAITE buffer $(10 \mathrm{mM}$ Tris- $\mathrm{HCl}, \mathrm{pH} 7.4 ; 25 \mathrm{mM}$ sucrose; $75 \mathrm{mM}$ sorbitol; 100 $\mathrm{mM} \mathrm{KCl} ; 10 \mathrm{mM} \mathrm{K} \mathrm{HPO}_{4} ; 0.05 \mathrm{mM}$ EDTA; $5 \mathrm{mM} \mathrm{MgCl}_{2} ; 1 \mathrm{mg} / \mathrm{mL} \mathrm{BSA}$ ) either untreated, treated with $20 \mathrm{mM} \mathrm{NaCl}$ and $100 \mu \mathrm{M} \mathrm{CaCl}_{2}$ or with $20 \mathrm{mM} \mathrm{NaCl}, 100 \mu \mathrm{M} \mathrm{CaCl}_{2}$ and $10 \mu \mathrm{M}$ CGP-37157. Substrates and inhibitors were then successively added: $5 \mathrm{mM}$ glutamate and $5 \mathrm{mM}$ malate $(\mathrm{GM}), 1 \mu \mathrm{M}$ rotenone, $10 \mathrm{mM}$ succinate, $2.5 \mu \mathrm{g} / \mathrm{mL}$ antimycin $\mathrm{A}, 10 \mathrm{mM}$ N,N,N',N'tetramethyl-p-phenylenediamine and $10 \mathrm{mM}$ sodium azide. Oxygen consumption rate (OCR) was obtained by calculating the slope after each treatment. Values for specific complex input were acquired from the subtraction of substrate less specific inhibitor rates.

\section{Mitochondrial membranes isolation and complex activity measurement}

Mitochondrial membranes from BAECs were obtained after freezing-thawing isolated mitochondria, and OXPHOS enzyme activity was measured as described ${ }^{37}$, using $50 \mu \mathrm{g}$ per sample. Briefly, rotenone-sensitive NADH-ubiquinone $\mathrm{Q}_{1}$ oxidoreduction (complex I activity) was measured by changes in absorbance at $340 \mathrm{~nm}$. Succinate dehydrogenase (complex II) activity was recorded in a buffer containing mitochondrial membranes, succinate and 2,6dichlorophenol-indophenol (DCPIP) by changes in absorbance at $600 \mathrm{~nm}$. Rotenone-sensitive NADH-cytochrome $c$ activity (complex I+III activity) was measured by changes in absorbance at $550 \mathrm{~nm}$ after NADH addition. Antimycin A-sensitive succinate-cytochrome $c$ activity (complex II + III activity) was calculated after measuring changes in absorbance at $550 \mathrm{~nm}$. ROS production in CII+CIII activity was monitored by fluorescence of dichlorofluorescein probe (DCF). Antimycin A-sensitive ubiquinone 2- cytochrome $c$ activity (complex III activity) was measured following changes in absorbance at $550 \mathrm{~nm}$. Glycerol-3-Phosphate dehydrogenase (G3PD)+III activity was measured as for CII+III activity but with glycerol-3-Phosphate $(10 \mathrm{mM})$ as electron donor. 


\section{Measurement of mitochondrial $\mathrm{Na}^{+}$}

In some experiments, cells were preincubated with $10 \mu \mathrm{M}$ CGP-37157 for $30 \mathrm{~min}$. Then, cells were treated with normoxia, or hypoxia $\left(1 \% \mathrm{O}_{2}\right)$ for $10 \mathrm{~min}$. Mitochondria were isolated on ice and resuspended in milliQ water. The samples were split, one part incubated with benzofuran isophthalate tetra-ammonium salt (SBFI) and the other was used to quantify protein amount by BCA. For SBFI measurements, a calibration curve was used in every measurement and the fluorescence recorded at $340 \mathrm{~nm} / 380 \mathrm{~nm}$ and emission $520 \mathrm{~nm}$ in a FLUOstar Omega Microplate Reader (BMG Labtech).

\section{Fluorescence recovery after photobleaching (FRAP)}

Cells were transfected with pDsRed2-Mito Vector (Clontech). Growing media was changed for $\mathrm{HBSS}+\mathrm{Ca} / \mathrm{Mg}+\mathrm{Glucose} \pm 10 \mu \mathrm{M}$ CGP-37157 and the plate placed into a Leica SP-5 confocal microscope, an automated stage for live imaging and a thermostated hypoxic cabinet. The planes were focused for image capture and images were taken with a $63 \times$ objective with $13 \times$ zoom.

Samples were excited with an argon/krypton laser using the $514 \mathrm{~nm}$ line and emission was detected in the 565-595 $\mathrm{nm}$ range. Images were collected using TCS software (Leica). MitoRFP was scanned five times and then bleached using 15 scans at $40 \%$ laser power. To image the recovery of fluorescence intensity after photobleaching, we recorded 60 scans every $1 \mathrm{~s}$. FRAP in normoxia was performed at $20 \% \mathrm{O}_{2}$ and $5 \% \mathrm{CO}_{2}$, the chamber was then switched to $1 \% \mathrm{O}_{2}$ and $5 \% \mathrm{CO}_{2}$ and after 25 min FRAP was performed in hypoxia.

\section{Fluorescence quenching}

Medium of BAECs was changed for $\mathrm{HBSS}+\mathrm{Ca} / \mathrm{Mg}+$ Glucose with $50 \mathrm{nM} 10 \mathrm{~N}$-nonyl acridine orange (NAO). In some experiments $10 \mu \mathrm{M}$ CGP-37157 was added. The plate placed into a Leica SP-5 confocal microscope, an automated stage for live imaging and a thermostated hypoxic cabinet. The planes were focused for image capture and images were taken with a $63 \times$ objective with 13x zoom.

Samples were excited with an argon/krypton laser using the $488 \mathrm{~nm}$ line and emission was detected in the 515-535 $\mathrm{nm}$ range. Images were collected using TCS software (Leica). NAO was scanned two times and then bleached using 15 scans at 20\% laser power. To image the quench of fluorescence intensity after photobleaching, we recorded 30 scans every 0.372 s. Quenching recording in normoxia was performed at $20 \% \mathrm{O} 2$ and $5 \% \mathrm{CO} 2$, the chamber was then switched to $1 \% \mathrm{O} 2$ and 5\% CO2 and the quench again recorded after 15 min in hypoxia.

\section{Fourier-transform infrared spectroscopy (FT-IR)}

Phosphatidylcholine liposomes were prepared using the thin film hydration method followed by extrusion with filters of decreasing diameter $(400,200 \text { and } 100 \mathrm{~nm})^{45}$. Lipids were hydrated with water with minimal metal impurities (Optima ${ }^{\mathrm{TM}} \mathrm{LC} / \mathrm{MS}$ Grade, Fisher Chemical). Liposomes were concentrated by filtration up to $72 \mathrm{mg} / \mathrm{mL}$ of lipid content. Lipid concentration was determined through the Rouser assay ${ }^{46}$. Sodium salt was mixed and incubated at $37{ }^{\circ} \mathrm{C}(2 \mathrm{~h})$ with

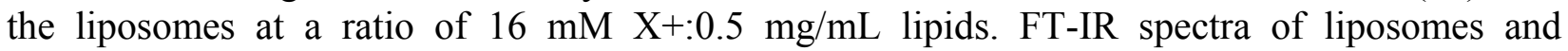
liposomes incubated with alkali metals were collected afterwards with a Nicolet 6700 FT-IR spectrometer (Thermo Scientific) in transmission mode in liquid samples. All experiments were performed in triplicate. 


\section{Inductively-Coupled Plasma Mass Spectroscopy (ICP-MS)}

Phosphatidylcholine liposomes were mixed and incubated at $37^{\circ} \mathrm{C}(2 \mathrm{~h})$ with sodium chloride salt

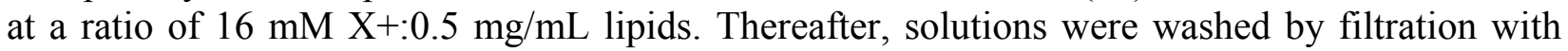
Optima $^{\mathrm{TM}}$ LC/MS Grade water and digested with $\mathrm{HNO} 3$ acid $([\mathrm{Na}+]=13$ ppt, Nitric Acid Optima, for Ultra Trace Elemental Analysis, Fisher Chemicals). The number of alkali cations bound to each lipid within the liposomes, after 3 washing steps, was determined with ICP-MS iCAP-Q from Thermo Scientific equipped with a collision/reaction cell and Kinetic Energy Discrimination (KED). Lipid concentration was determined through the Rouser assay ${ }^{46}$. All experiments were performed in triplicate.

\section{Transmission electron microscopy}

EM of cells and isolated mitochondria were performed as described ${ }^{47}$ and thin sections were imaged on a Tecnai-20 electron microscope (Philips-FEI). Quantification of area and frequency of electron dense spots was performed manually and using Image J software.

\section{Transmission electron microscopy and energy-dispersive X-ray spectroscopy analysis (TEM-EDX)}

Cell samples were fixed using 4\% formaldehyde (EMS) and $2.5 \%$ glutaraldehyde (Sigma) in 0.1 M HEPES for 4 hours at $4^{\circ} \mathrm{C}$. Samples were post-fixed 1 hour at RT in a $2 \%$ osmium tetroxide (EMS) and 3\% potassium ferrocyanide (Sigma) solution. Cell preparations were dehydrated through graded acetone series and embedded in Spurr's Low Viscosity embedding mixture (EMS). Ultrathin sections $(70 \mathrm{~nm})$ were then mounted on formvar coated copper grids and stained with lead citrate. Samples were examined on a Jeol JEM 1400 with SDD (Silicon Drift Detector) and elemental content estimation was performed under 80.000-100.000 magnification and using 50-100 seconds lifetime acquisition. Data on oxygen $(\mathrm{O})$, carbon $(\mathrm{C})$, lead $(\mathrm{Pb})$ and calcium $(\mathrm{Ca})$ were collected from the TEM-EDX analysis. $\mathrm{C}, \mathrm{O}$ and $\mathrm{Pb}$ were used as controls.

\section{Measurement of redox state of ubiquinone}

The ratio of the oxidized and reduced forms of ubiquinone $(\mathrm{CoQ})$ were measured as described ${ }^{48-}$ 50. $10 \mu \mathrm{M}$ CGP-37157 or antimycin A was added $30 \mathrm{~min}$ before the experiment and maintained throughout. HUVECs with or without CGP-37157 were subjected to $10 \mathrm{~min}$ of hypoxia $\left(1 \% \mathrm{O}_{2}\right)$ or normoxia. Antmicyin A was used in normoxic cells as a control. Plates were washed three times with ice-cold PBS and the cells were transferred to ice-cold tubes, which were subsequently centrifuged at $2000 \mathrm{~g}$ for $5 \mathrm{~min}$ at $4^{\circ} \mathrm{C}$. Cell pellets were resuspended in $95 \mu 1$ of PBS from which $91.5 \mu \mathrm{l}$ were taken and mixed with $3.5 \mu 114.3 \mu \mathrm{M}$ 2-mercaptoethanol. $5 \mu 110 \mu \mathrm{M}$ coenzyme Q6 were added as internal standard. Then, $330 \mu 1$ 1-propanol were added, the sample vortexed for 30 $\mathrm{s}$, incubated for $3 \mathrm{~min}$ at RT, vortexed again for $20 \mathrm{~s}$ and centrifuged at 14,000 g for $5 \mathrm{~min}$. $100 \mu \mathrm{l}$ of the supernatant was immediately injected into a 166-126 HPLC system (Beckman-Coulter) equipped with an UV/Vis detector (System Gold R 168, Beckman-Coulter) and an electrochemical (Coulochem III ESA) detector. Separation was carried out in a $15 \mathrm{~cm}$ Kromasil $\mathrm{C} 18$ column (Scharlab, Spain) at $40^{\circ} \mathrm{C}$ with a mobile phase $20 \mathrm{mM}$ ammonium acetate $\mathrm{pH} 4.4$ in methanol (solvent A) and $20 \mathrm{mM}$ ammonium acetate $\mathrm{pH} 4.4$ in propanol (solvent B). A gradient method was used with a 85:15 solvent mixture (A:B ratio), flow rate of $1.2 \mathrm{~mL} / \mathrm{min}$, as the starting conditions. The mobile phase turns to a 50:50 A:B ratio starting at minute 6 and completed in minute 8 , flow rate decreases in parallel to $1.1 \mathrm{~mL} / \mathrm{min}$. After $20 \mathrm{~min}$ (run time), the columns are re-equilibrated to the initial conditions for three additional minutes. UV-spectrum was used to identify the different forms of ubiquinone (oxidized CoQ10 with maximum absorption at $275 \mathrm{~nm}$ ) and ubiquinol (reduced $\mathrm{CoQ}, \mathrm{CoQ}_{10} \mathrm{H}_{2}$, with maximum absorption at 290 
$\mathrm{nm})$ using specific standards. Quantification was carried out with electrochemical detector reading s(channel 1 set to $-700 \mathrm{mV}$ and channel 2 set to $+500 \mathrm{mV}$, conditioning guard cell after injection valve).

\section{Electron spin resonance (ESR) of lipid vesicles}

ESR spectroscopy is particularly useful to investigate the rotational dynamics of labeled molecules in solutions or membranes. The rotational dynamics timescale, $\tau_{c}$, is inversely related to the fluidity of the microenvirement of the probe. ESR experiments were carried out with a 9.5GHz ESR Bruker spectrometer (RSE Bruker EMX). $2 \mathrm{mM}$ DOPC unilamelar vesicles were obtained by ultrasonication. The spin labels $(0.25 \% \mathrm{~mol}$ of total lipid composition) were added to the dry lipids before vesicle formation to obtain a symmetrical distribution of probes in the two leaflets. We used 5-doxyl palmitoyl PC (5-Doxyl PC), 12-doxyl palmitoyl PC (12-Doxyl PC) and 16-doxyl palmitoyl PC (16-Doxyl PC). The different position of the probe along the alkyl chain determines the local motional profiles in the three main regions of the lipidic bilayer, near the polar head group (5-Doxyl PC), at the middle region of the hydrophobic chain (12-Doxyl PC) or at the end of the hydrophobic chain (16-Doxyl PC). The ESR spectrum of 5-Doxyl PC incorporated into the lipidic membranes made of DOPC shows an anisotropic (slow) motion whereas those of both 12-Doxyl PC and 16-Doxyl PC reflects an isotropic motion of the acylchain. The $\tau_{c}$ of 5-Doxyl PC is proportional to the outermost separation between the spectral extrema, $2 \mathrm{~A}_{\max }$, whereas the $\tau \mathrm{c}$ of 12-Doxyl PC and 16-Doxyl PC were calculated by the semiempirical formula established ${ }^{51}, \tau_{\mathrm{c}}=6.5 \cdot 10^{-10} \Delta \mathrm{B}_{0}\left(\sqrt{\frac{\mathrm{h}_{0}}{\mathrm{~h}_{-1}}}-1\right)$ where $\Delta \mathrm{B}_{0}$ is the width of the central line in Gauss $(G)$, and $h_{0}$ and $h_{-1}$ are the heights of the mid- and high-field lines respectively. $2 \mathrm{~A}_{\max }, \Delta \mathrm{B}_{0}, \mathrm{~h}_{0}$ and $\mathrm{h}_{-1}$ were obtained from the first derivative of each absorption spectrum.

\section{Steady-state fluorescence emission anisotropy of DPH or TMA-DPH incorporated into lipid vesicles}

Mixtures of 1-3 mg of egg phosphatidylcholine (PC; Avanti Polar Lipids Inc., Birmingham, AL, USA), cardiolipin (CL; Avanti Polar Lipids Inc., Birmingham, AL, USA) to a final molar PC:CL molar ratio of 4:1 (when present), and the fluorescent lipid probes diphenyl-1,3,5-hexatriene (DPH; Sigma-Aldrich) or 1-(4(trimetylamonium)-phenyl)-6-diphenyl-1,3,5-hexatriene (TMA$\mathrm{DPH}$; Sigma-Aldrich), at a lipid/probe molar ratio of $1 / 100$, were dissolved in $200 \mu \mathrm{L}$ of chloroform:methanol (2:1) and dried under flow of nitrogen for at least $60 \mathrm{~min}$. The resulting dry lipid film was then resuspended in $1.5 \mathrm{~mL}$ of MilliQ-degree water and vortexed vigorously for 1 min. This suspension of multilamelar vesicles was then incubated for one hour at $37{ }^{\circ} \mathrm{C}$. Large unilamelar vesicles were then prepared by extrusion of this lipid dispersion through polycarbonate filters of controlled $100 \mathrm{~nm}$ diameter pore size. These vesicles were maintained at $37{ }^{\circ} \mathrm{C}$ for no more than $3 \mathrm{~h}$ before being used ${ }^{52}$. The steady-state fluorescence emission anisotropy experiments were carried out on a SLM Aminco 8000C spectrofluorimeter (SLM Aminco, Urbana, IL, USA), as previously described ${ }^{53}$. Fluorescence anisotropy (r) of DPH or TMA-DPH incorporated into the liposomes described above was recorded at $37{ }^{\circ} \mathrm{C}$ using excitation and emission wavelengths of 350 or 356 and 452 or $451 \mathrm{~nm}$, respectively.

\section{Measurement of pulmonary artery contraction}

Third division branches of the pulmonary arteries (PAs) were isolated from male Wistar rats and mounted in a wire myograph. Contractile responses were recorded as reported ${ }^{50}$. The chambers were filled with Krebs buffer containing (in $\mathrm{mM}$ ) $\mathrm{NaCl} 118, \mathrm{KCl} 4.75, \mathrm{NaHCO}_{3} 25, \mathrm{MgSO}_{4} 1.2$, $\mathrm{CaCl}_{2} 2.0, \mathrm{KH}_{2} \mathrm{PO}_{4} 1.2$ and glucose 11 , maintained at $37^{\circ} \mathrm{C}$ and aerated with $21 \% \mathrm{O}_{2}, 5 \% \mathrm{CO}_{2}$, $74 \% \mathrm{~N}_{2}$ gas $\left(\mathrm{pO}_{2} 17-19 \mathrm{kPa}\right)$. After an equilibration period of $30 \mathrm{~min}$, PAs (internal diameter 
$300-400 \mu \mathrm{m})$ were distended to a resting tension corresponding to a transmural pressure of 2.66 $\mathrm{kPa}$. Preparations were initially stimulated by raising the $\mathrm{K}^{+}$concentration of the buffer (to 80 $\mathrm{mM}$ ) in exchange for $\mathrm{Na}^{+}$. Vessels were washed three times and allowed to recover. Then, each vessel was exposed to two hypoxic challenges $\left(95 \% \mathrm{~N}_{2}, 5 \% \mathrm{CO}_{2} ; \mathrm{pO}_{2}=2.6-3.3 \mathrm{kPa}\right)$, the second one after 40 min incubation with vehicle (control) or CGP-37157 $(30 \mu \mathrm{M})$.

\section{Statistics}

Data are presented as mean \pm SEM. Normality and homoscedasticity tests were carried out before applying parametric tests. For comparison of multiple groups, we performed one-way analysis of variance (ANOVA) followed by Tukey's or Bonferroni's tests for all the groups of the experiment. For comparison of two groups, we used Student's two-tailed t-test; when the data did not pass the normality test, we used a non-parametric t-test (Mann-Whitney U test). Linear correlation was estimated calculating the Pearson correlation coefficient. Variations were considered significant when the $\mathrm{p}$ value was less than 0.05 . Statistical analysis was performed with SigmaPlot 11.0 or GraphPad Prism 8 software.

\section{Data availability}

The data that support the findings of this study are available from the corresponding author upon reasonable request. 


\section{Acknowledgments}

We thank Dr. Mariusz Kowalewski (Institute of Veterinary Anatomy, UZH) for allowing us the use of the microscope for live cell imaging. We thank Alejandra Alfuzzi, Javier Prieto, Alejandro Mellado (IIS-IP) for collaboration in experiments, Esther Fuertes-Yebra (IIS-IP), for technical assistance, Dr. María Eugenia Soriano and Dr. Federico Caicci (Univ. Padova) for performing electron microscopy, Dr. Judith Langer from CIC biomaGUNE for fruitful discussion and support with the IR spectroscopy measurements, Dr. María Cano and Prof. Antonio G. García (IIS-IP and UAM), Dr. Carlos Rueda and Prof. Jorgina Satrústegui (CMBSO, UAM-CSIC), Prof. Mike Murphy (MRC \& Univ. Cambridge), Dr. Israel Sekler (Ben-Gurion University), Dr. Ilka Wittig (Goethe Universität), Dr. José Miguel Mancheño (IQFR, CSIC) and Dr. Alberto Pascual and Prof. José López-Barneo (IBIS, US-CSIC) for helpful discussions, and Dr. Luis del Peso (UAM) and Prof. Francisco Sánchez-Madrid (IIS-IP and UAM) for their support. This research has been financed by Spanish Government grants (partially funded by the European Union FEDER/ERDF) CSD2007-00020 (RosasNet, Consolider-Ingenio 2010 programme to A.M.R. and J.A.E.), CP07/00143, PS09/00101, PI12/00875, PI15/00107, and RTI2018-094203-B-I00 (to A.M.R), CP12/03304 and PI15/01100 (to L.M.), SAF2016-77222-R (to A.C.), SAF2015-65633-R (to J.A.E), SAF2013-32223 (to M.G.L.) and SAF2017-84494-2-R (to J.R.C.), by the European Union (ITN GA317433 to J.A.E. and MC-CIG GA304217 to R.A.P.), by a grant from the Comunidad de Madrid (B2017/BMD-3727, to A.C.), by a grant from the Fundación Domingo Martínez (to M.G.L. and A.M.R.), by grants from the Fundación BBVA (to R.A.P. and J.R.C.), by the Programa Red Guipuzcoana de Ciencia, Tecnología e Información 2018-CIEN-000058-01 (J.R.C) and from the Basque Government under the ELKARTEK Program (Grant No. KK2019/bmG19 to J.RC.), by Swiss National Science Foundation (SNF) grant 310030_124970/1 to A.B, by a travel grant from the Instituto de Investigación Sanitaria Princesa (to P.H.-A.) and by the COST actions TD0901 (HypoxiaNet) and BM1203 (EU-ROS). CNIC is supported by the ProCNIC Foundation and is a Severo Ochoa Center of Excellence (Spanish Government award SEV2015-0505). CIC biomaGUNE is supported by the María de Maeztu Units of Excellence Program from the Spanish Government (MDM-2017-0720). P.H.-A. was recipient of a predoctoral FPU fellowship from the Spanish Government, E.N. is recipient of a predoctoral FPI fellowship from the Universidad Autónoma de Madrid (UAM), and A.M.-R., L.M. and J.E. are supported by the I3SNS or "Miguel Servet" programmes (ISCIII, Spanish Government; partially funded by FEDER/ERDF).

\section{Author contributions}

P.H.A. and A.M.R. conceived the idea. P.H.A., J.A.E. and A.M.R. designed the study. P.H.A, C.C.F. E.R. and T.O. performed the bulk of the experiments and analysed the data. A.V.L.V. performed EDX experiments. L.M. and A.C. carried out PA contractility experiments. P.H.A., A.C.R., J.C.R.A. and P.N. analysed the redox state of ubiquinone. P.H.A., R.A.P. and J.A.E. analysed mitochondrial complex and supercomplex activities. E.N., E.P., A.P.A., J.E., M.G.L., E.R., J.D.C.G., T.V.P., A.I.A., D.T., P.J. and J.W.E. helped with crucial experimental procedures. P.J. and J.W.E. provided NCLX KO fibroblasts. I.L.R. performed ESR experiments with liposomes. A.M.P. performed experiments with DPH or TMA-DPH labelled liposomes, P.H.A., S.C.R. and J.R.C. performed IR and ICP-MS experiments of $\mathrm{Na}^{+}$-phospholipid interaction. A.B., J.A.E. and A.M.R. supervised the study and P.H.A., J.A.E. and A.M.R. wrote the manuscript.

The authors declare no competing financial interest. 


\section{Extended Data}

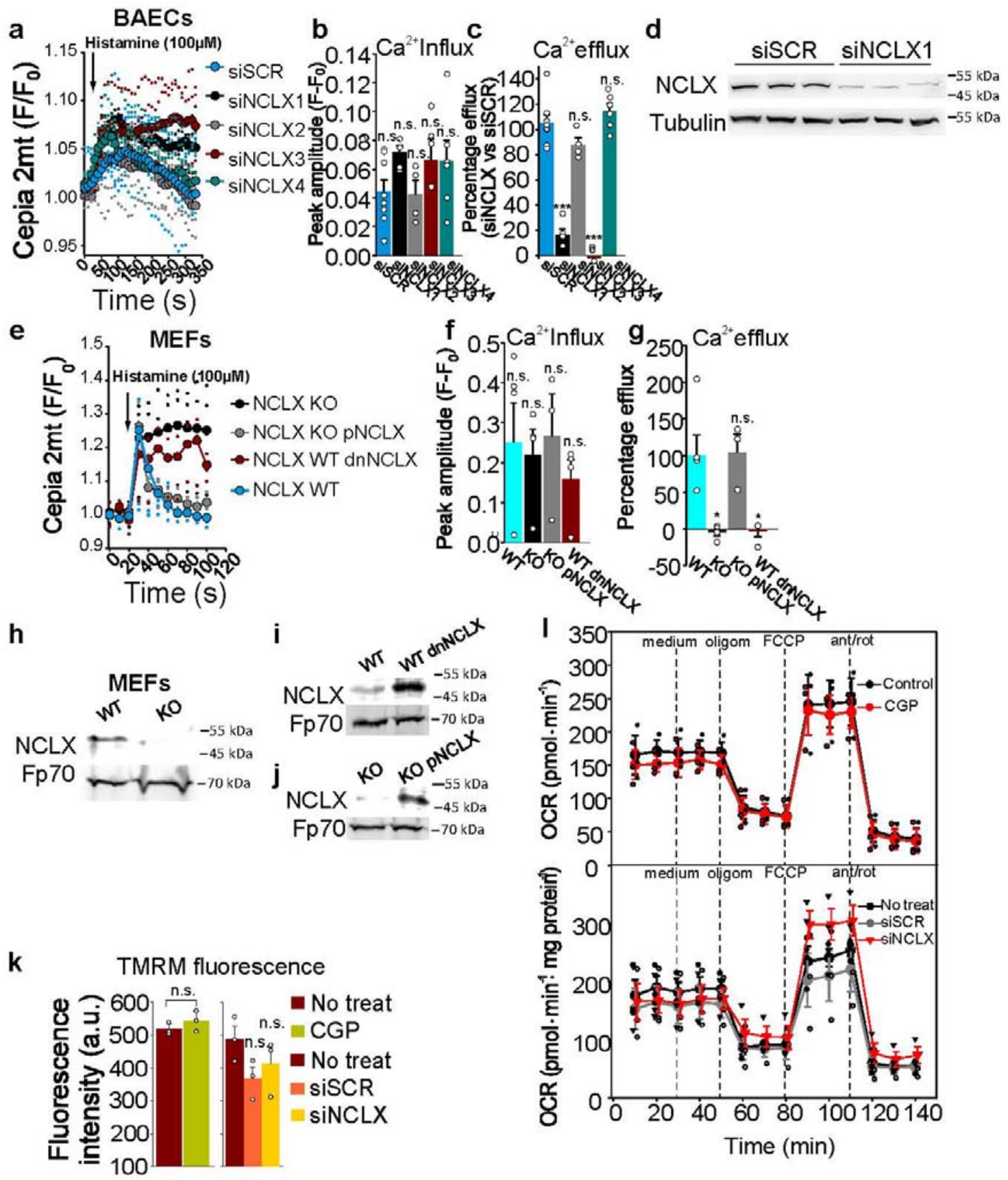

Extended Data Fig. 1. Interference of Slc8b1 affects NCLX function, but inhibition of NCLX has no effect on general mitochondrial function. a-c, Assessment of the effect of different siRNAs against Slc8b1 (siNCLX) on mitochondrial $\mathrm{Ca}^{2+}$ influx and efflux rates in BAECs transfected with the mitochondria-directed $\mathrm{Ca}^{2+}$ reporter protein Cepia $2 \mathrm{mt}(\mathrm{n} \geq 4)$, after addition of histamine; a, mean traces plotted as fluorescence change relative to initial fluorescence $\left(\mathrm{F} / \mathrm{F}_{0}\right)$; $\mathbf{b}$, Mitochondrial $\mathrm{Ca}^{2+}$ peak amplitude (calculated from the highest value of 
fluorescence minus the first basal value of fluorescence); c, Percentage of mitochondrial $\mathrm{Ca}^{2+}$ efflux (calculated from the highest value of fluorescence minus the lowest value of fluorescence after histamine application, relative to percental siSCR values). d, Assessment of NCLX protein amount after interference in whole-cell extracts from BAECs, by Western blot (representative image of $\mathrm{n}=3$ ).. e-g, Mitochondrial $\mathrm{Ca}^{2+}$ influx and efflux rates in $\mathrm{WT}, \mathrm{KO}, \mathrm{KO}+\mathrm{pNCLX}$ or $\mathrm{WT}+\mathrm{dnNCLX}$ MEFs transfected with Cepia $2 \mathrm{mt}(\mathrm{n} \geq 4)$ after addition of histamine, as in a-c. h-j, Assessment of NCLX protein amount in MEFs mitochondrial extracts by Western blot (representative images of $n=3$ ). $\mathbf{k}$, Mitochondrial membrane potential in BAECs measured with $20 \mathrm{nM}$ TMRM in non-quenching mode $(\mathrm{n}=3)$. l, Oxygen consumption rate (OCR) in BAECs with NCLX inhibition by $10 \mu \mathrm{M}$ CGP-37157 (upper panel, $\mathrm{n}=3$ ), or NCLX interference with siNCLX1 (lower panel, $\mathrm{n}=4$ ). 


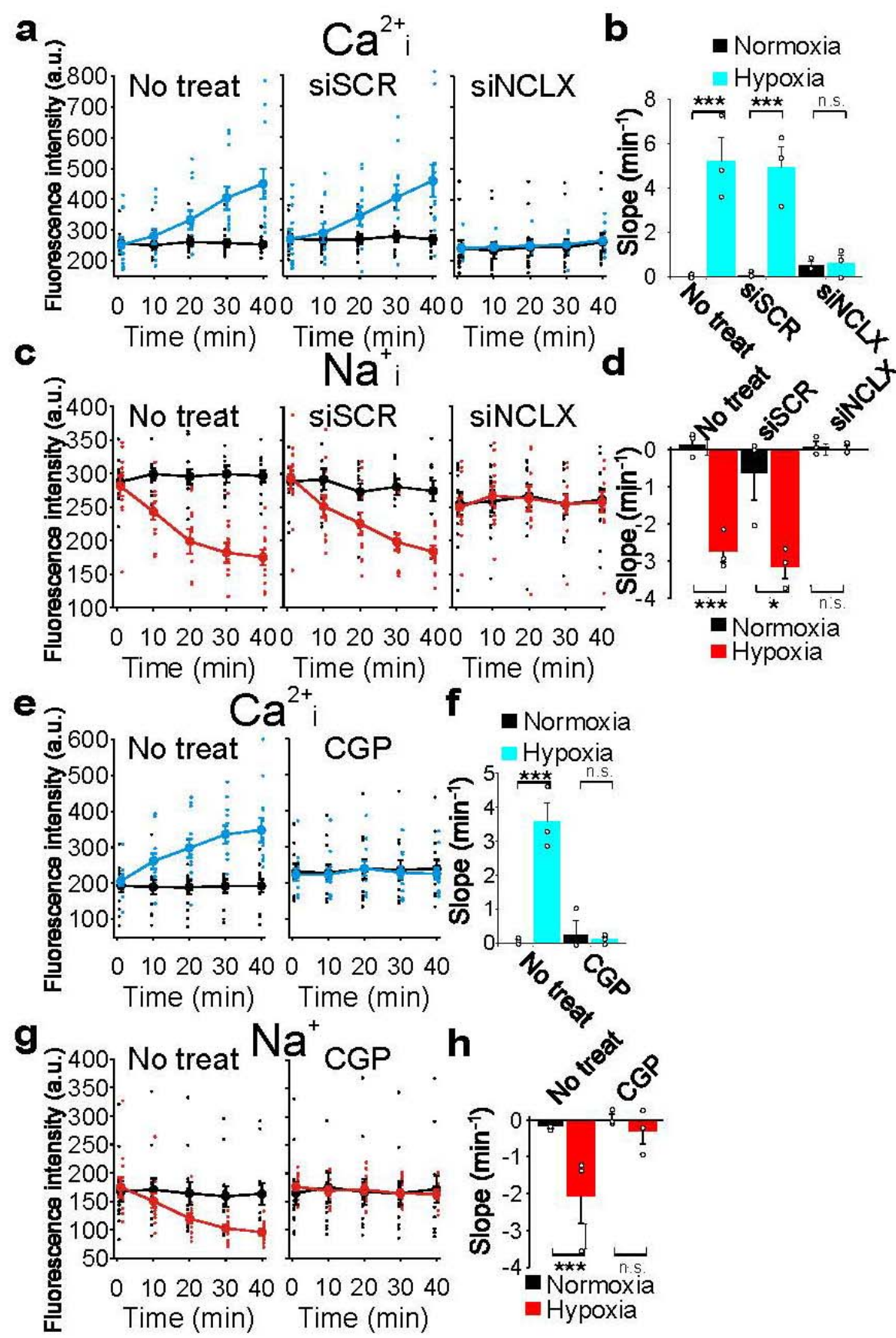

Extended Data Fig. 2. Hypoxia activates $\mathrm{Na}^{+} / \mathrm{Ca}^{2+}$ exchange through NCLX. Cytosolic $\mathrm{Ca}^{2+}$ $\left(\mathrm{Ca}^{2+}{ }_{\mathrm{i}}\right)$ or $\mathrm{Na}^{+}\left(\mathrm{Na}^{+}{ }_{\mathrm{i}}\right)$ were measured by live imaging fluorescence microscopy with Fluo-4 AM or CoroNa Green, respectively, in normoxia or acute ischemia $\left(2 \% \mathrm{O}_{2}\right.$ without glucose $)$. a-d, BAECs not treated (No treat) or transfected with siSCR or siNCLX. e-h, BAECs treated or not with the NCLX inhibitor CGP-37157 (10 $\mu \mathrm{M})$. a,c,e,g, time-course traces; b,d,f,h, slopes. n=3. * $\mathrm{p}<0.05, * * \mathrm{p}<0.01, * * * \mathrm{p}<0.001$, n.s. not significant (Student's t-test). 

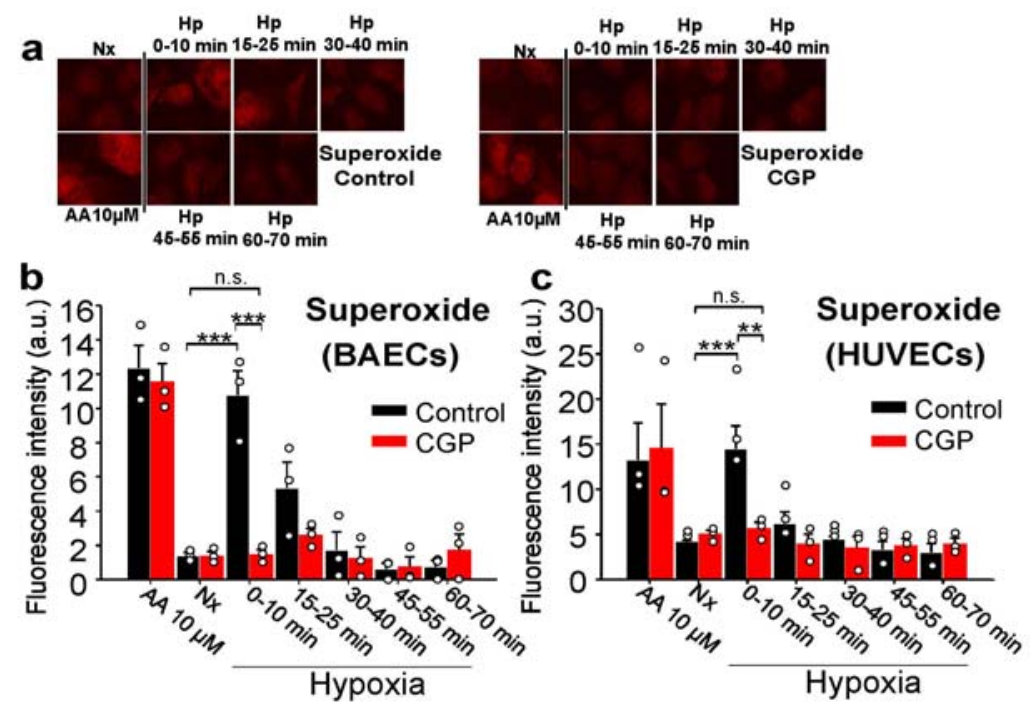

d

Cytosolic $\mathrm{H}_{2} \mathrm{O}_{2}$
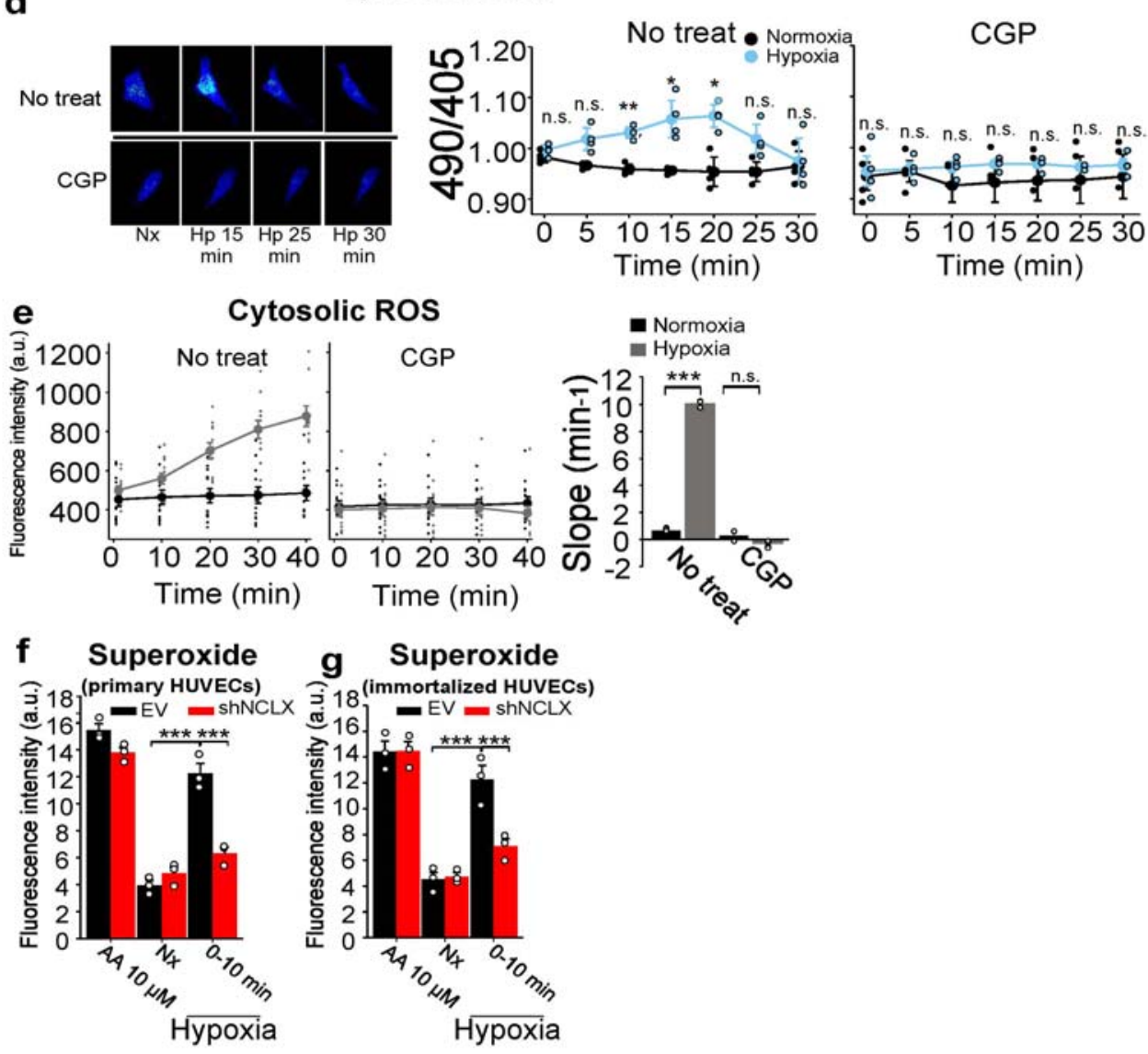

\section{Extended Data Fig. 3. Inhibition of NCLX prevents the increase in ROS production}

triggered by hypoxia. a-c, Superoxide detection by fluorescence microscopy after incubation with DHE in 10-min time windows in normoxia $(\mathrm{Nx})$ or hypoxia $\left(1 \% \mathrm{O}_{2}\right)$; AA $=$ antimycin $\mathrm{A}$. ab, BAECs with $10 \mu \mathrm{M}$ CGP-37157, images of one experiment and mean intensity of three independent experiments; c, HUVECs with $10 \mu \mathrm{M}$ CGP-37157, mean intensity of three independent experiments. d, Detection of $\mathrm{H}_{2} \mathrm{O}_{2}$ by live confocal microscopy in CytoHyPer- 
transfected BAECs either non-treated or treated with CGP in normoxia $(\mathrm{Nx})$ or acute hypoxia $\left(1 \% \mathrm{O}_{2}, \mathrm{Hp}\right)$; representative images and time-course traces as mean of three independent experiments. e, Detection of ROS by live fluorescence microscopy with DCFDA in normoxia or transition from normoxia to acute hypoxia $\left(2 \% \mathrm{O}_{2}\right)$; time-course traces and slopes, mean of three independent experiments. f-g, Superoxide detection by fluorescence microscopy with DHE in normoxia $(\mathrm{Nx})$ or 10 min hypoxia $\left(1 \% \mathrm{O}_{2}\right)$ or AA in primary (f) or immortalized (g) HUVECs; mean of three independent experiments. Two-tailed Student's t-test for pairwise comparisons and one-way ANOVA and Tukey's test for multiple comparisons n.s. not significant, ${ }^{*} \mathrm{p}<0.05$, $* *<\mathrm{p} 0.01, * * * \mathrm{p}<0.001$. b-c, statistical comparisons shown only for Nx vs 0-10 groups. 
a

BAECs

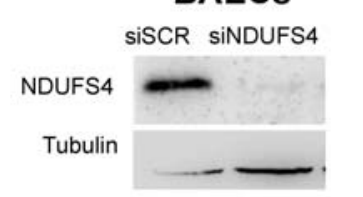

b

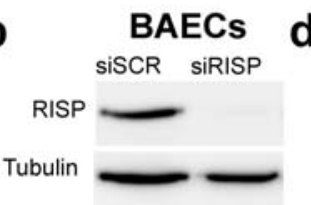

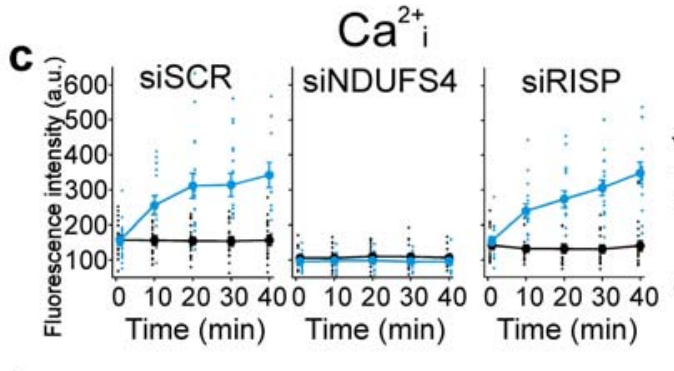
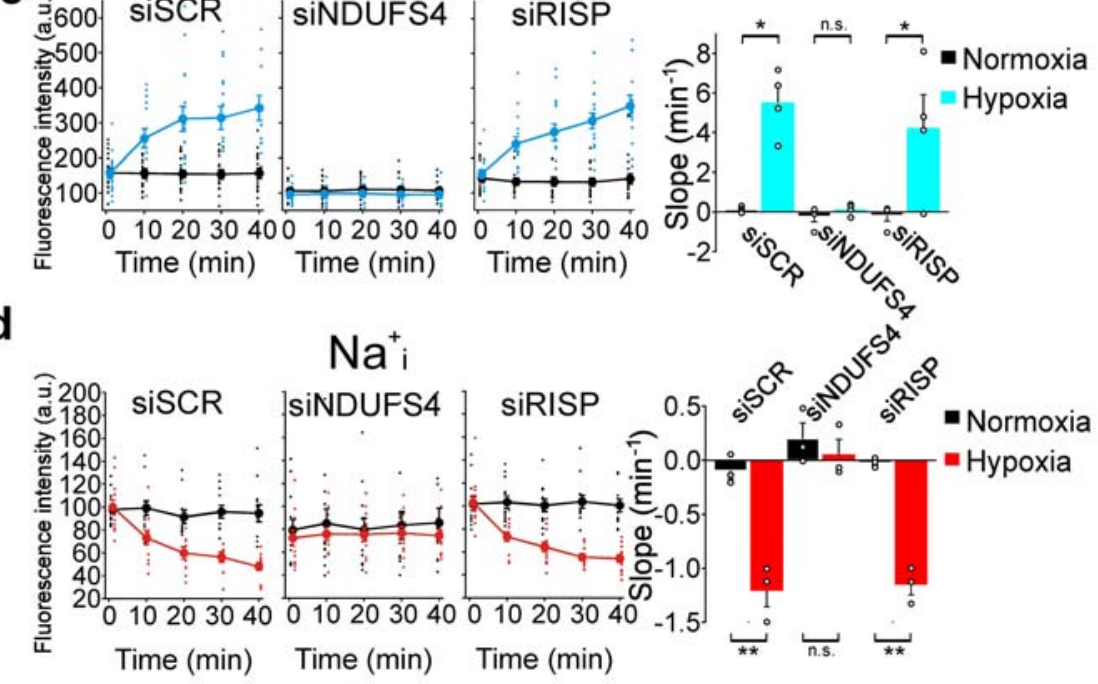

$\mathrm{Na}^{+}$
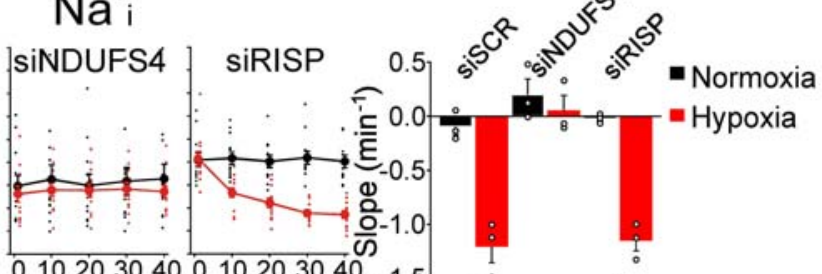

e

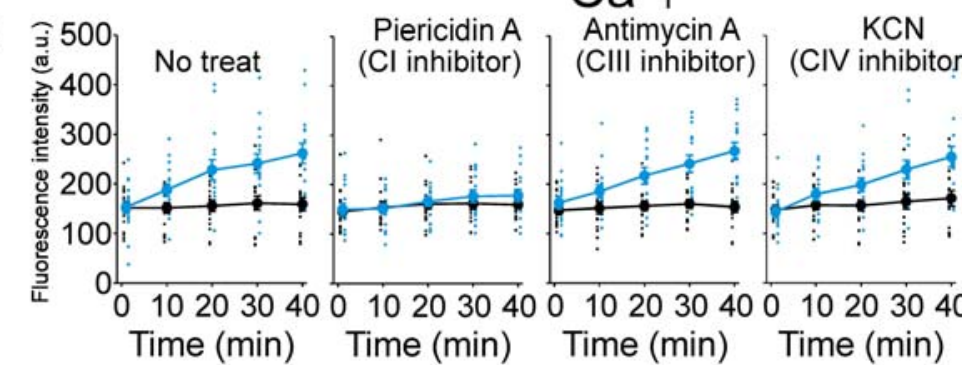

$\mathrm{Na}^{+}{ }_{\mathrm{i}}$
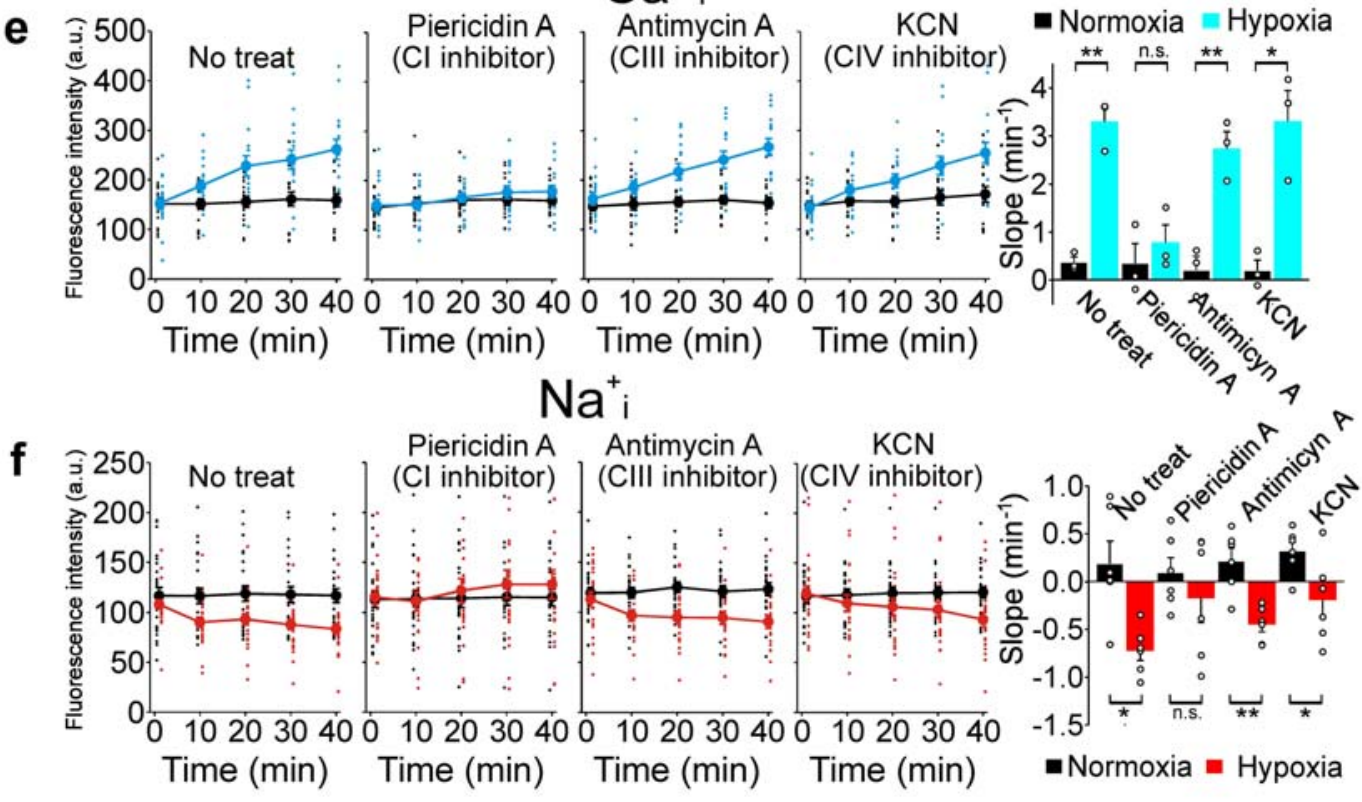

\section{Extended Data Fig. 4. NCLX activation in acute hypoxia depends on mitochondrial complex}

I. a, b, Assessment of interference of subunits of CI (NDUFS4) or CIII (RISP) by Western blot in whole-cell extracts from BAECs. c, d, Cytosolic $\mathrm{Ca}^{2+}\left(\mathrm{Ca}^{2+}{ }_{\mathrm{i}}\right)$ or $\mathrm{Na}^{+}\left(\mathrm{Na}^{+}{ }_{\mathrm{i}}\right)$ were measured by live imaging confocal microscopy with Fluo-4 AM or CoroNa Green, respectively, in normoxia or acute hypoxia $\left(1 \% \mathrm{O}_{2}\right)$, time-course traces and slopes, $\mathrm{n}=3\left(\mathrm{Ca}^{2+}{ }_{\mathrm{i}}\right), \mathrm{n}=4\left(\mathrm{Na}^{+}\right)$. e-f, Effect of OXPHOS inhibitors on cytosolic $\mathrm{Ca}^{2+}(\mathbf{e}, \mathrm{n}=3)$ or $\mathrm{Na}^{+}(\mathbf{f}, \mathrm{n}=4)$ measured as in $\mathbf{c}$, d. Two-tailed Student's t-test. n.s. not significant, $* \mathrm{p}<0.05, * *<\mathrm{p} 0.01$. 

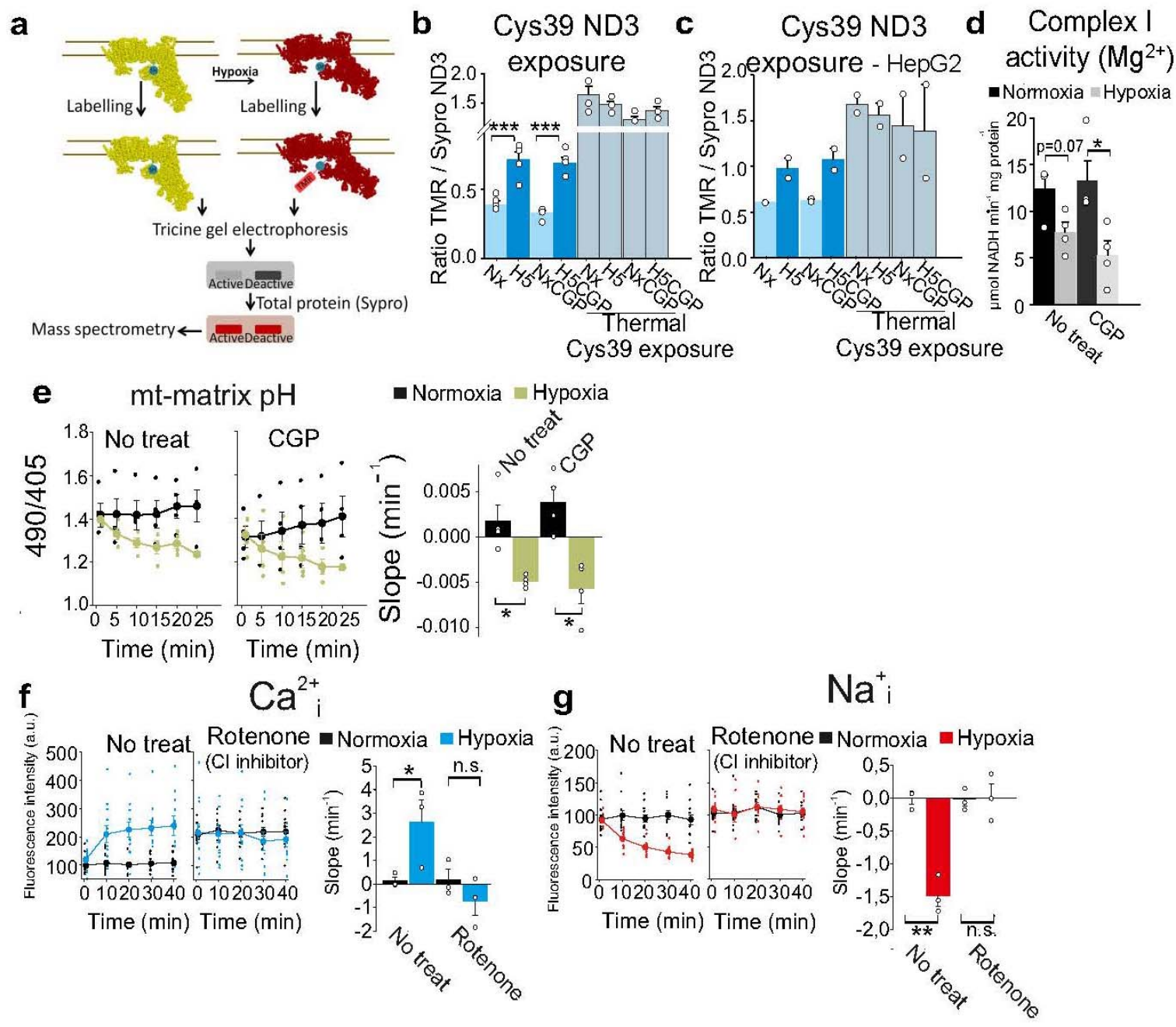

\section{Extended Data Fig. 5. Acute hypoxia promotes A/D transition in complex I independently of}

NCLX activity. a-c, ND3. Cys39 exposure, which reflects the D state of CI, measured as the ratio between TMR signal (Cys39 labelling) and Sypro Ruby staining (total protein for the ND3 band, identified by mass spectrometry ${ }^{11}$ ). Thermal deactivation is used as a positive control of CI D state. a, scheme of the technique ${ }^{11}$; BAECs $(\mathbf{b}, \mathrm{n}=4)$ or HepG2 $(\mathbf{c}, \mathrm{n}=2)$ exposed to normoxia $(\mathrm{Nx}), 5 \mathrm{~min}$ of hypoxia $\left(1 \% \mathrm{O}_{2}, \mathrm{H} 5\right)$, normoxia with CGP-37157 (NxCGP), 5 min of hypoxia with CGP-37157 (H5CGP). d, Complex I reactivation rate measured in the presence of $\mathrm{Mg}^{2+}$ in isolated mitochondrial membranes from BAECs subjected to normoxia, $10 \mathrm{~min}$ of hypoxia (1\% $\mathrm{O}_{2}$ ) and NCLX inhibition with CGP-37157 (n=4). e, Mitochondrial matrix acidification induced by acute hypoxia $\left(1 \% \mathrm{O}_{2}\right)^{11}$ and the effect of NCLX inhibition in BAECs transfected with mitosypHer, time-course traces and slopes $(n=4)$. $\mathbf{f}-\mathbf{g}$, Effect of the CI inhibitor rotenone on cytosolic $\mathrm{Ca}^{2+}$ (f) or cytosolic $\mathrm{Na}^{+}$(g) measured by live confocal microscopy with Fluo-4 AM or CoroNa Green, respectively, in BAECs in normoxia or acute hypoxia $\left(1 \% \mathrm{O}_{2}\right), \mathrm{n}=3$. Two-tailed Student's t-test for pairwise comparisons and one-way ANOVA with Tukey's test for multiple comparisons. n.s. not significant, $* \mathrm{p}<0.05, * *<\mathrm{p} 0.01, * * * \mathrm{p}<0.001$. 

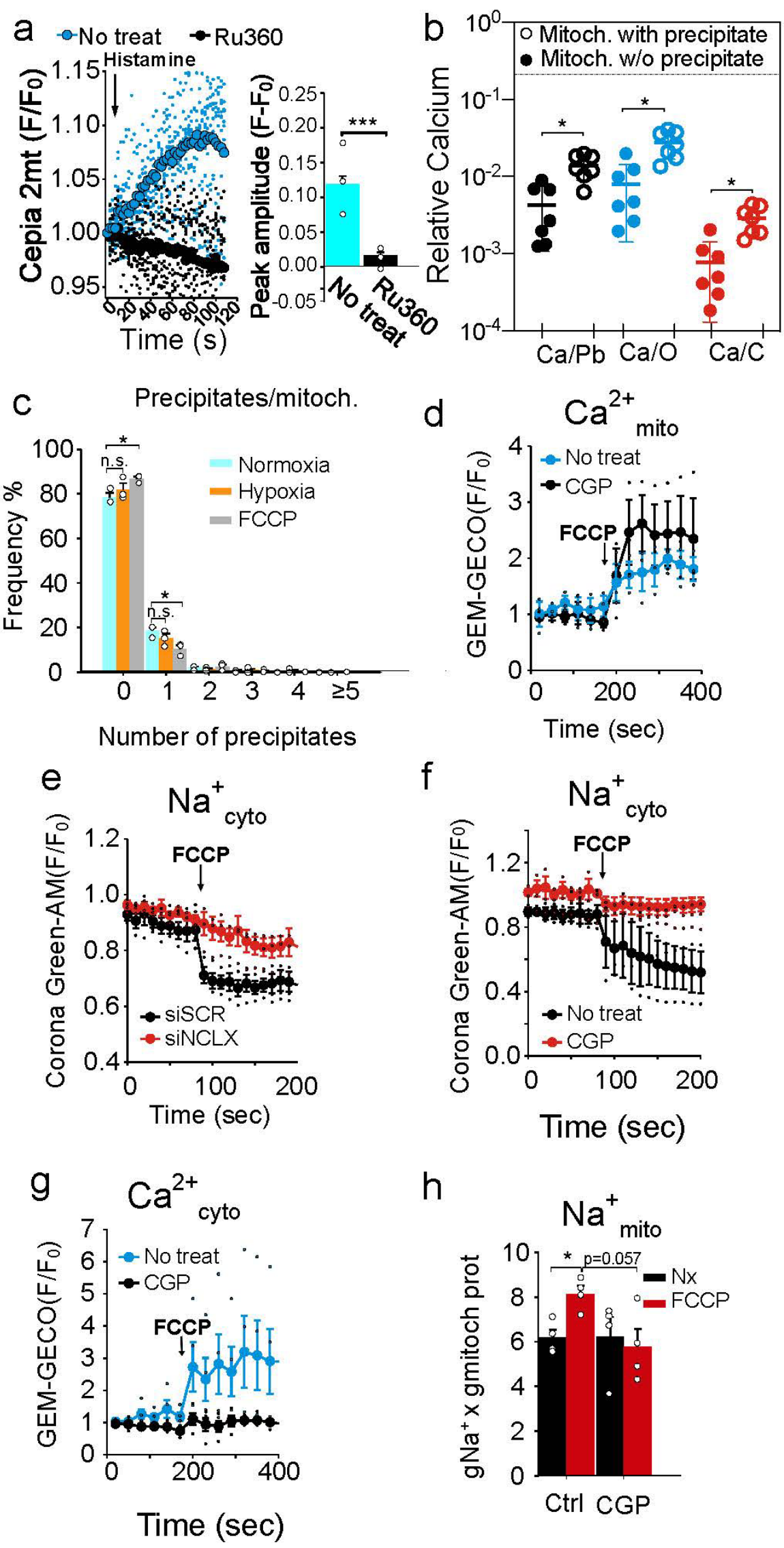

h

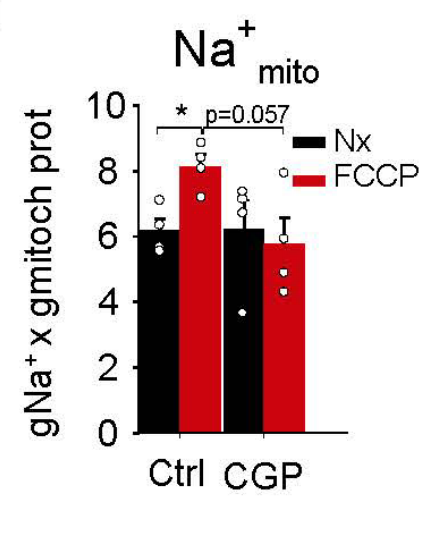

Extended Data Fig. 6. Mitochondrial matrix acidification promotes mitochondrial $\mathrm{Na}^{+} / \mathrm{Ca}^{2+}$ exchange via NCLX. a, Assessment of the effect of $1 \mu \mathrm{M}$ Ru360 on mitochondrial $\mathrm{Ca}^{2+}$ influx 
rates in BAECs transfected with the mitochondria-directed $\mathrm{Ca}^{2+}$ reporter protein Cepia $2 \mathrm{mt}(\mathrm{n}=3)$, after addition of histamine. Left, mean traces plotted as fluorescence change relative to initial fluorescence $\left(\mathrm{F} / \mathrm{F}_{0}\right)$; right, mitochondrial $\mathrm{Ca}^{2+}$ peak amplitude. b, TEM-EDX determination of calcium element $(\mathrm{Ca})$ versus carbon $(\mathrm{C})$, oxygen $(\mathrm{O})$ and lead $(\mathrm{Pb})$ content in regions of mitochondria with (empty circles) or without (filled circles) electron dense spots. c, Frequency of calcium phosphate precipitates per mitochondrion in BAECs, seen by transmission electron microscopy during normoxia (741 mitochondria), $10 \mathrm{~min}$ of hypoxia (1\% $\mathrm{O}_{2} ; 619$ mitochondria) or 30 min with $1 \mu \mathrm{M}$ FCCP (393 mitochondria), sum of three independent experiments. d, Mitochondrial $\mathrm{Ca}^{2+}$ measured by live cell confocal microscopy in BAECs transfected with mitoGEM-GECO, either non-treated or treated with CGP-37157, before and after addition of $1 \mu \mathrm{M}$ FCCP. e, f, Cytosolic $\mathrm{Na}^{+}$measured by live cell confocal microscopy with CoroNa Green in BAECs transfected with siSCR or siNCLX (e), non-treated BAECs or treated with CGP-37157 (f), before and after addition of $1 \mu \mathrm{M}$ FCCP. f, Cytosolic $\mathrm{Ca}^{2+}$ measured by live cell confocal microscopy in BAECs transfected with cyto-GEM-GECO, either non-treated or treated with CGP-37157, before and after addition of $1 \mu \mathrm{M}$ FCCP. $\mathbf{h}$, Mitochondrial $\mathrm{Na}^{+}$measured by SBFI fluorimetry in mitochondria isolated from non-treated BAECs (Con) or treated with CGP-37157, FCCP or both. Fluorescence signal relative to starting signal (F/F0), n=3 (except $\mathbf{h}, \mathrm{n}=4)$. Oneway ANOVA with Tukey's test. n.s. not significant, * ${ }^{*}<0.05$. 


\section{a}
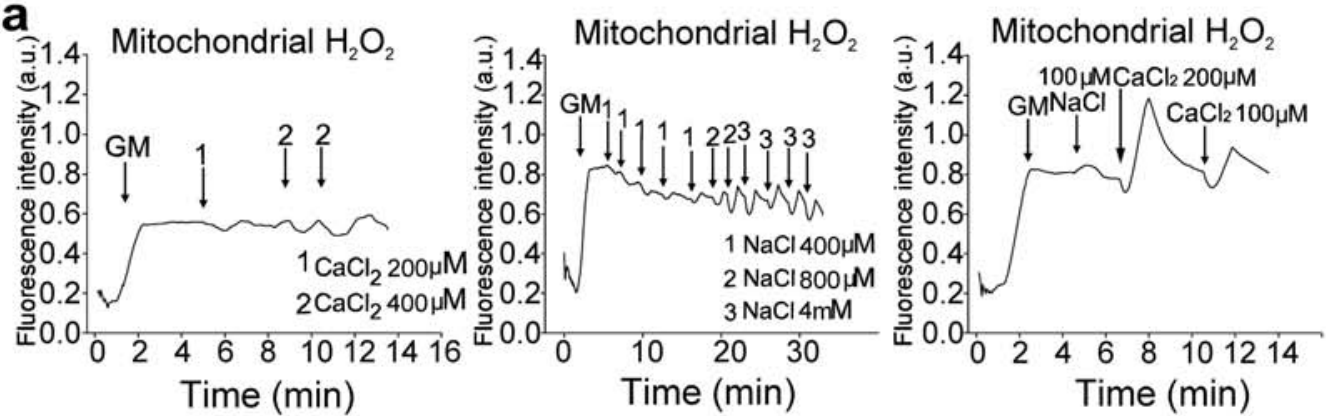

b 2 -respiration ClV-respiration

d e
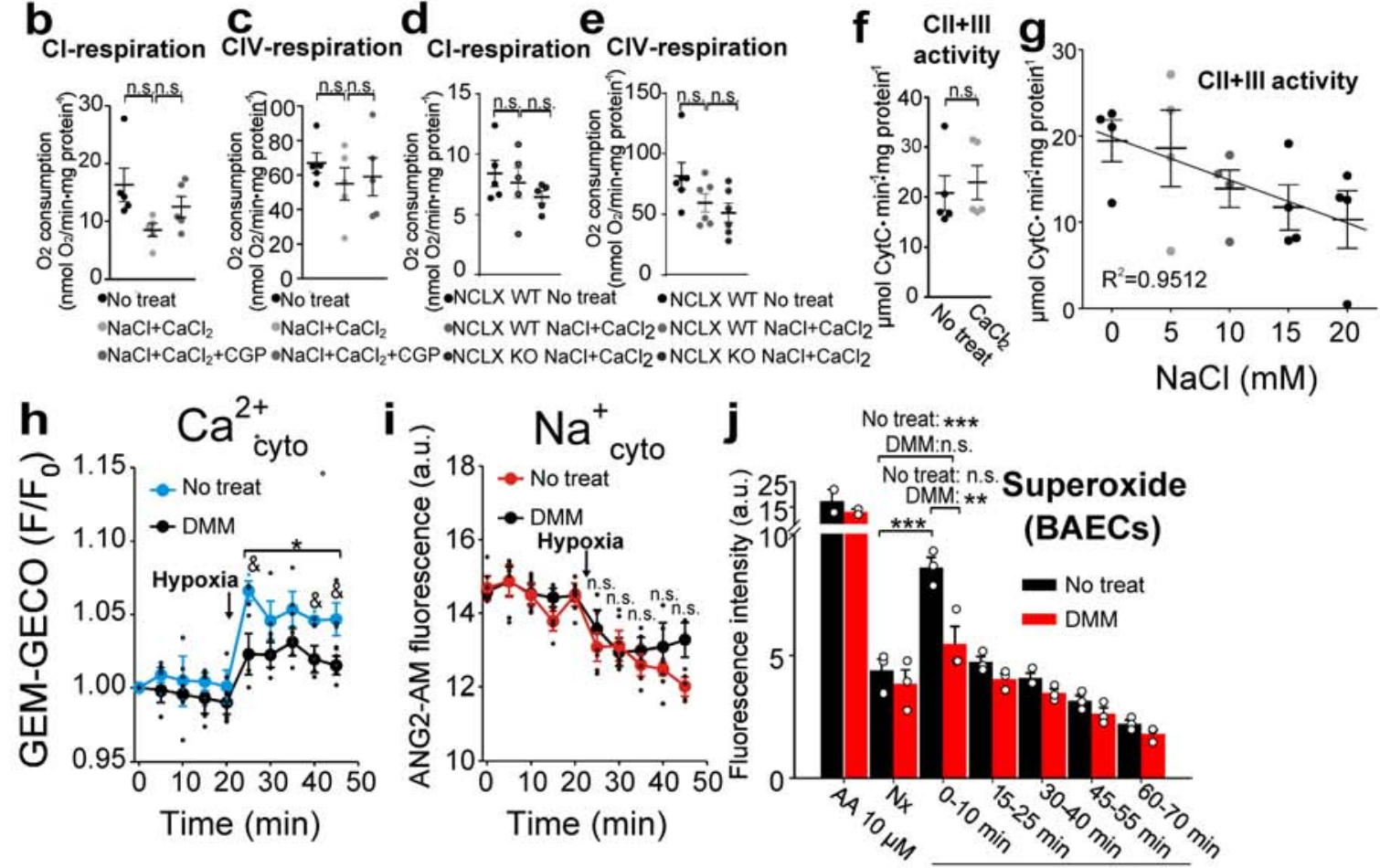

CIII activity

Time (min)

Hypoxia

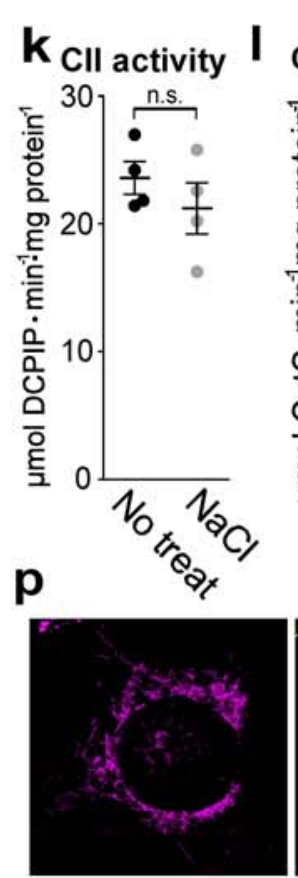

CoronaGreen

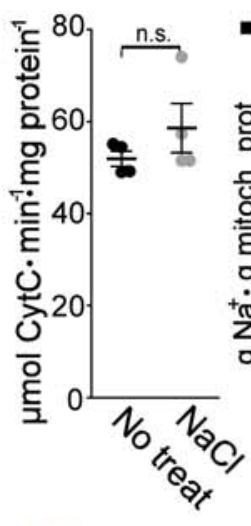

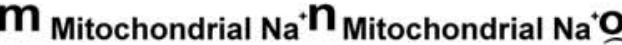
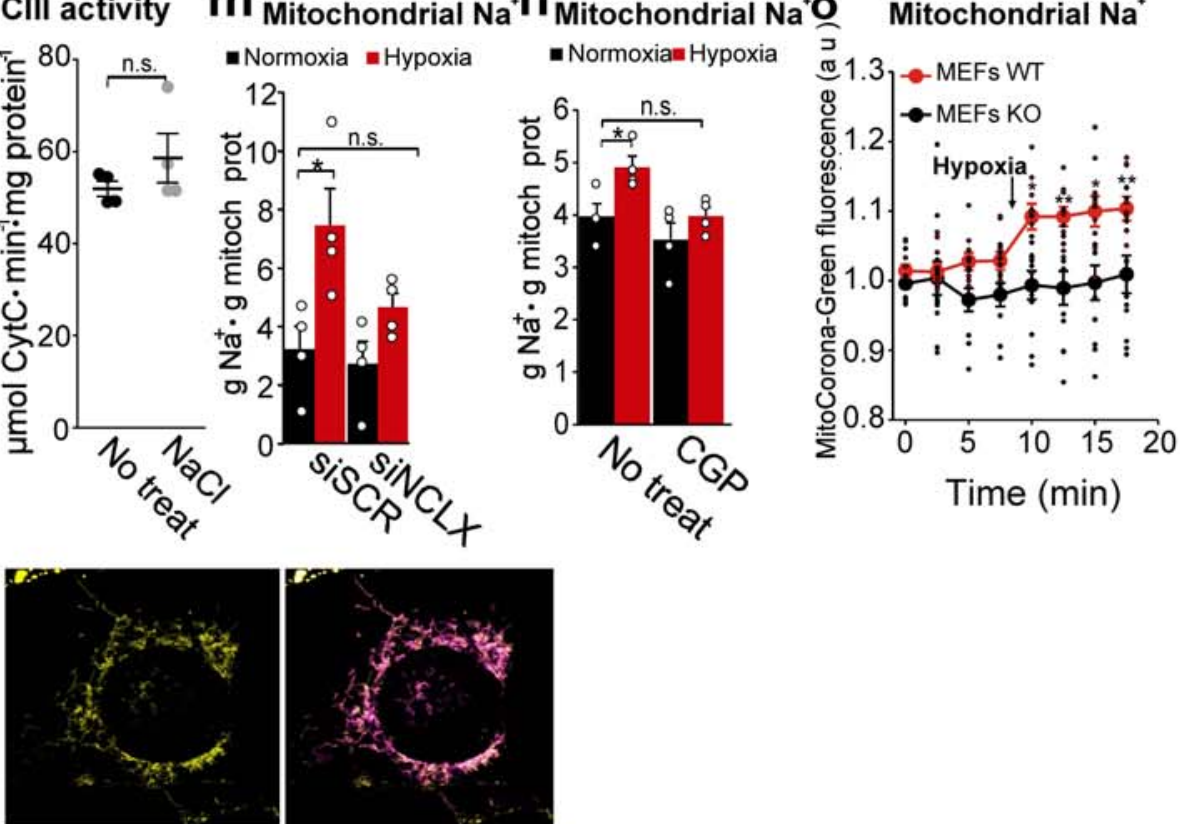

TMRM
- Normoxia =Hypoxia
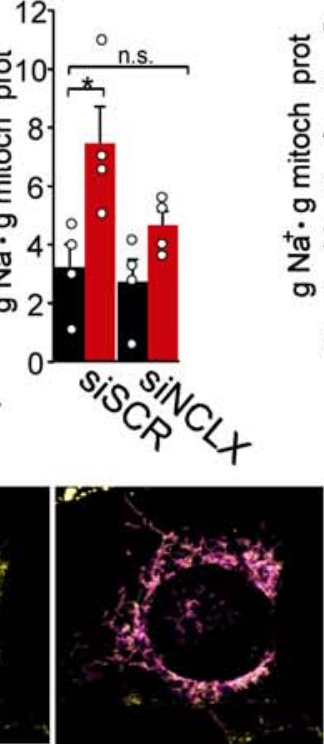

Merge 


\section{Extended Data Fig. 7. Mitochondrial $\mathrm{Na}^{+}$import decreases OXPHOS and produces ROS. a,} Effect of $\mathrm{NaCl}$ and/or $\mathrm{CaCl}_{2}$ additions on $\mathrm{H}_{2} \mathrm{O}_{2}$ production detected with Amplex Red in isolated rat heart mitochondria $(500 \mu \mathrm{g})$ respiring after addition of glutamate/malate (GM) in KCl-EGTA buffer. Representative traces of five independent experiments. b-e, Effect of NCLX activation by $10 \mathrm{mM} \mathrm{NaCl} / 0.1 \mathrm{mM} \mathrm{CaCl}_{2}$ on glutamate/malate- (b, d) or TMPD-based (c, e) OCR in isolated coupled mitochondria from BAECs (b-c) or MEFs (d-e) (n=5). f, g, Effect of $0.1 \mathrm{mM} \mathrm{CaCl} 2$ $(\mathrm{n}=5)$ or $\mathrm{NaCl}(\mathrm{n}=4)$ additions on CII+III activity in isolated mitochondrial membranes from BAECs. h, Cytosolic $\mathrm{Ca}^{2+}$ measured by live cell confocal microscopy of BAECs transfected with cyto-GEM-GECO, either non-treated (No treat) or treated with $10 \mu \mathrm{M}$ DMM during normoxia and hypoxia $\left(1 \% \mathrm{O}_{2} ; \mathrm{n}=4\right)$. i, Cytosolic $\mathrm{Na}^{+}$measured by live cell confocal microscopy with ANG2-AM in non-treated BAECs (No treat) or treated with $10 \mu \mathrm{M}$ DMM during normoxia and hypoxia $\left(1 \% \mathrm{O}_{2} ; \mathrm{n}=4\right)$. j, Superoxide detection by fluorescence microscopy after incubation with DHE in 10-min time windows in non-treated BAECs (No treat) or treated with $10 \mu \mathrm{M} \mathrm{DMM}$ during normoxia $(\mathrm{Nx})$ or hypoxia $\left(1 \% \mathrm{O}_{2} ; \mathrm{n}=3\right)$. k-l, Effect of $10 \mathrm{mM} \mathrm{NaCl}$ addition on succinate dehydrogenase activity (k) or ubiquinone 2-cytochrome $c$ activity (l) from BAECs mitochondrial membranes $(\mathrm{n}=4)$. $\mathbf{m}-\mathbf{n}$, Effect of hypoxia $\left(1 \% \mathrm{O}_{2}\right)$ in $\mathrm{Na}^{+}{ }_{\text {mito }}$ content in BAECs transfected with siSCR, siNCLX (m) or treated with CGP-37157 (n) $(\mathrm{n}=4)$. o, Effect of hypoxia $\left(1 \% \mathrm{O}_{2}\right)$ in $\mathrm{Na}^{+}{ }_{\text {mito }}$ content in WT and KO MEFs, measured with CoroNa-Green adapted for mitochondrial loading and live fluorescence. $\mathbf{p}$, Representative images showing colocalization of Corona GreenAM and TMRM signals after application of a long incubation protocol for Corona Green AM. Student's t-test for pairwise comparisons and one-way ANOVA with Tukey's test for multiple comparisons. n.s. not significant, $* \mathrm{p}<0.05, * *<\mathrm{p} 0.01, * * * \mathrm{p}<0.001$. Student's t-test (No treat vs $\mathrm{DMM}):{ }^{\&} \mathrm{p}<0.05$. j, statistical comparisons shown only for $\mathrm{Nx}$ vs $0-10$ groups. Pearson correlation coefficient in $\mathbf{g}(\mathrm{R}=-0.9753)$. 


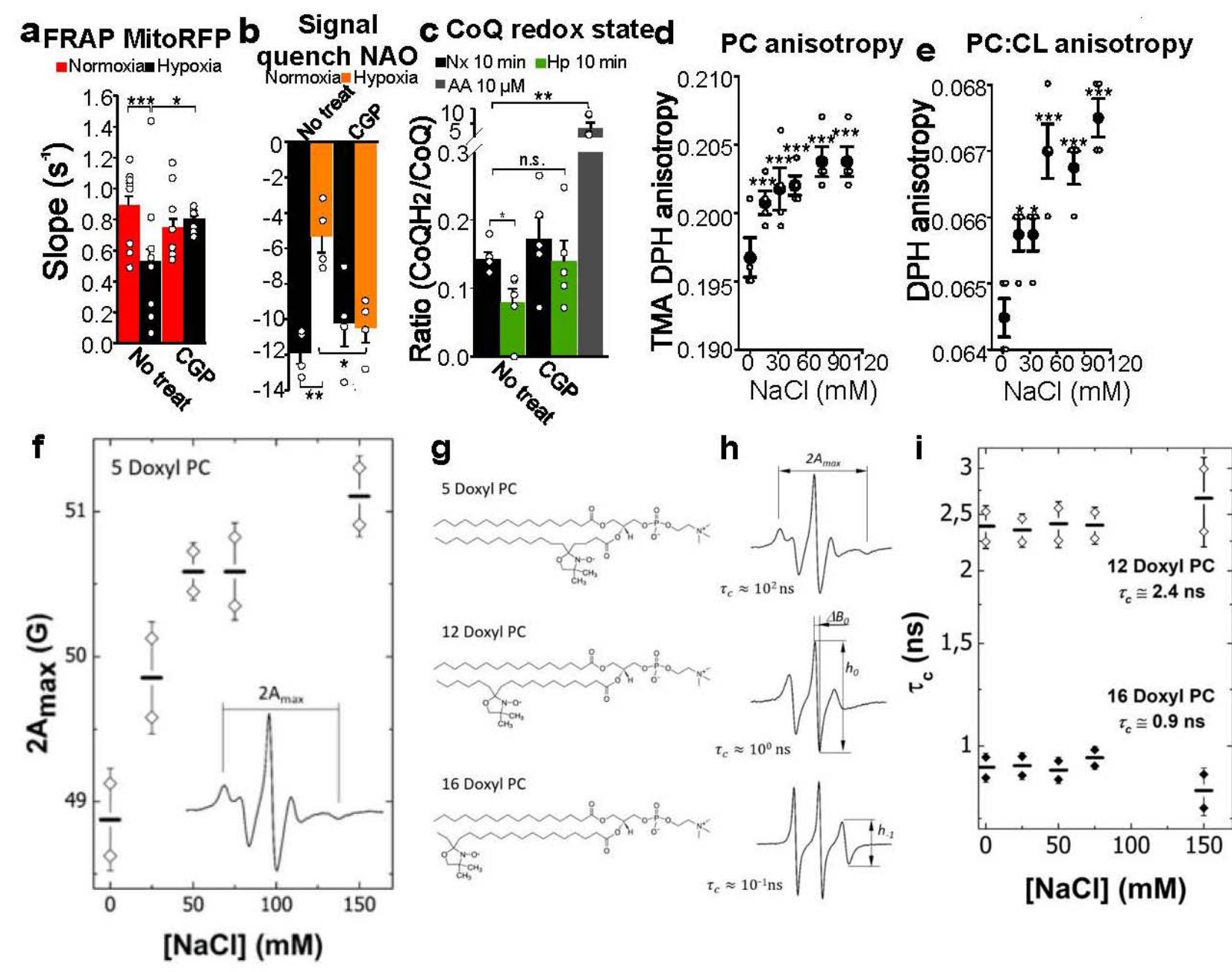

j

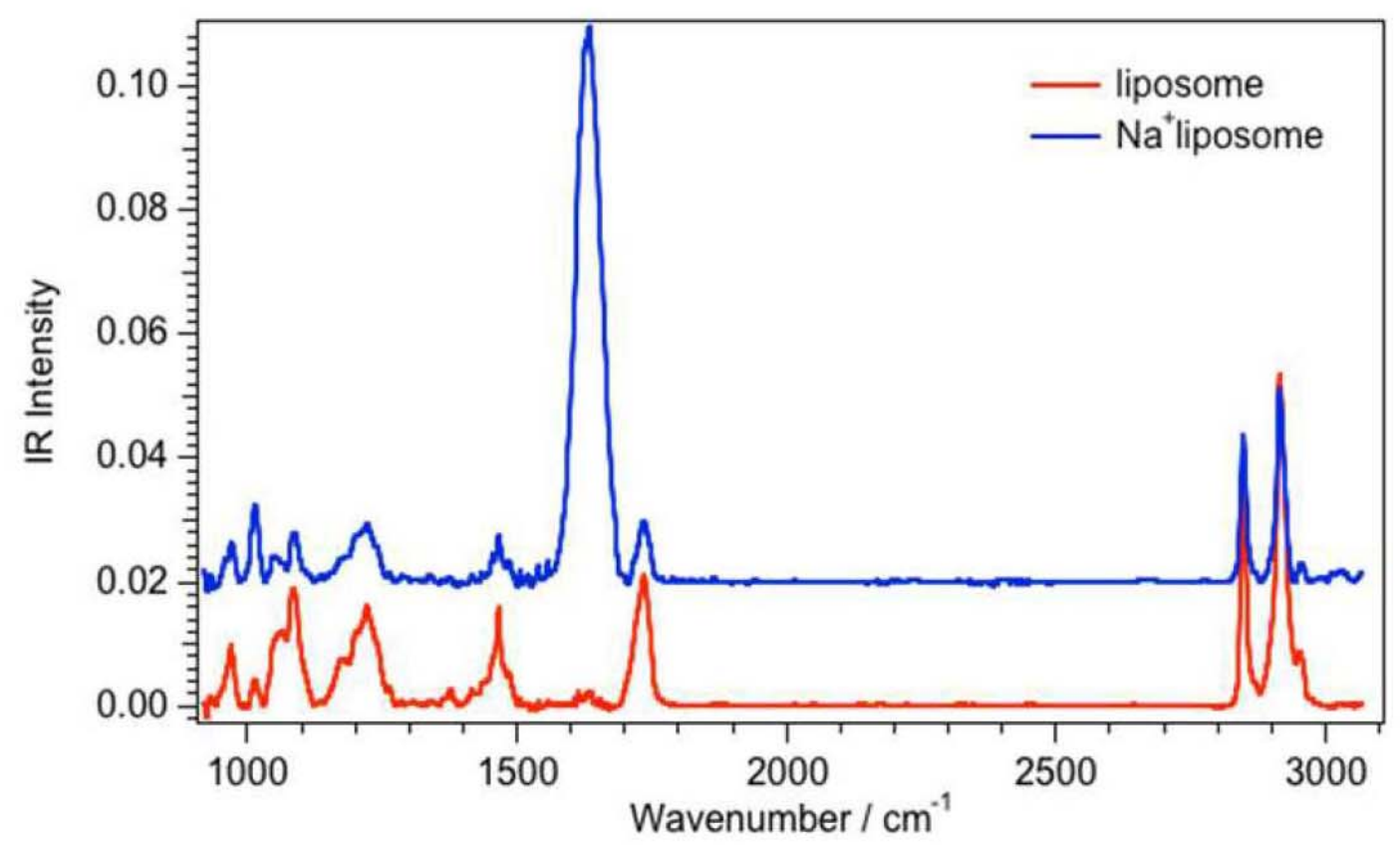

Extended Data Fig. 8. Mitochondrial $\mathrm{Na}^{+}$import decreases inner mitochondrial membrane fluidity. a, FRAP of BAECs expressing mitoRFP in normoxia or hypoxia for $20 \min \left(1 \% \mathrm{O}_{2} ; \mathrm{n} \geq\right.$ 
6 determinations from four independent experiments), with or without CGP-37157. b, NAO quench signal of BAECs exposed to normoxia or hypoxia for $15 \min \left(1 \% \mathrm{O}_{2} ; \mathrm{n}=5\right)$, with or without CGP-37157. c, CoQ 10 redox state of HUVECs subjected to normoxia (Nx), 10 min of hypoxia (Hp $10 \mathrm{~min} ; 1 \% \mathrm{O}_{2}$ ), or treated with $\mathrm{AA}, \mathrm{n}=4$. d-e, Anisotropy of phosphatydilcholine (PC) or PC:cardiolipin (PC:CL) liposomes treated with increasing concentrations of $\mathrm{NaCl}$ measured by TMA-DPH or DPH fluorescence. f-i, ESR spectra of 5-, 12- and 16- Doxyl PC in DOPC liposomes. 5-Doxyl PC exhibited an increased restricted motion (broadening of the hyperfine splitting, $2 A_{\max }$ ) as a function of the $\mathrm{NaCl}$ concentration whereas the correlation time for 12- and 16-Doxyl PC remained unchanged. f, Chemical structures of 5-, 12- and 16-Doxyl PC. g, Hyperfine splitting $\left(2 A_{\max }\right)$ of 5-Doxyl PC measured by ESR in PC liposomes treated with increasing concentrations of $\mathrm{NaCl}(\mathrm{n}=2)$. h, ESR of PC liposomes. $\mathbf{i}$, rotational times, $\tau_{c}$, of 12- and 16-Doxyl PC in PC liposomes treated with increasing concentrations of $\mathrm{NaCl}$ as measured by ESR ( $n=2)$. $\mathbf{j}$, IR spectroscopy absorption spectra of PC liposomes treated or not with $16 \mathrm{mM} \mathrm{NaCl}$. Student's t-test for pairwise comparisons and one-way ANOVA with Tukey's test for multiple comparisons. n.s. not significant, $* \mathrm{p}<0.05, * *<\mathrm{p} 0.01, * * * \mathrm{p}<0.001$. 